\title{
Coherent Proton Tunneling in Hydrogen Bonds of Isolated Molecules: Malonaldehyde and Tropolone
}

Richard L. Redington

1.1

Introduction

A series of articles reporting temperature dependent $\mathrm{H}$ and $\mathrm{D}$ isotope effects on the rates of certain enzymatic $\mathrm{H}$ transfer reactions show there is quantum tunneling by the $\mathrm{H}$ in these systems [1-7]. Theoretical considerations [4, 6, 7] infer vibrations within the enzyme-substrate complex, especially within the enzyme protein, develop transient geometrical configurations fomenting the tunneling process. Details of the promoting vibrations are still speculative, but it seems clear they work through transient reshaping of the potential energy surface (PES) barrier region, its width, energy maximum, overall contour, to provide "gated" impetus to $\mathrm{H}$ tunneling and to product escape.

Molecules hosting coherent $\mathrm{H}$ tunneling activity in the presence of complex vibrational structure are of immediate interest in the above context. They provide sharp data points probing specific vibrational couplings along the large amplitude tunneling coordinate, and they probe portions of the PES topography in detail. Studies on isotopomers and close chemical congeners provide clusters of data points reflecting systematic changes of the dynamical behavior. In this chapter research on the coherent tunneling properties of malonaldehyde, tropolone, and tropolone derivatives is surveyed. These are among the few currently known 1015 atom molecules showing clear-cut spectral doublet structures signifying coherent tunneling properties. Interestingly, the equal double-minimum global PESs for these molecules, notably for $\mathrm{S}_{0}$ tropolone, are also poised for demonstrations of tunneling activity by the heavy atoms. The presence of $\mathrm{H}$ tunneling in some enzymatic systems is newly recognized; tunneling processes by heavy atoms such as $\mathrm{C}, \mathrm{N}$, and $\mathrm{O}$ may also prove consequential. Zuev et al. [8] recently published low temperature rate data and theoretical-computational results showing the tunneling of $\mathrm{C}$ atom from a single quantum state during the ring expansion isomerization of 1-methycyclobutylfluorocarbene. 
As illustrated in Fig. 1.1, the tautomerizations of tropolone (TRN, $\mathrm{C}_{7} \mathrm{H}_{6} \mathrm{O}_{2}$ ) and malonaldehyde (MA, $\mathrm{C}_{3} \mathrm{H}_{4} \mathrm{O}_{2}$ ) involve an $\mathrm{O} \cdots \mathrm{HO} \rightarrow \mathrm{OH} \cdots \mathrm{O}$ transfer accompanied by shifts of the skeletal atom positions as the bond characters interchange. Spectroscopic experiments performed on low pressure gaseous samples of TRN and MA experience minimal disruptive interactions with the environment, and spectral doublet structures observed for both molecules unequivocally reveal coherent quantum tunneling. MA has 21 and TRN has 39 internal coordinates available for coupling into their tunneling processes. MA, with a developing experimental base [9-21], holds a computational edge over TRN which currently stands alone in its size group for its catalog of spectroscopic tunneling structures [22-39]. With 15 atoms TRN is reasonably expected to model elements of the intramolecular dynamical behavior occurring in much larger molecules, and it is amenable to high level experimental and theoretical studies. The topography of its PES has saddle-point (SP) energy barriers just low enough, and tautomer-to-tautomer heavy atom excursions that are just short enough, to produce numerous resolvable vibrational state-specific coherent tunneling splittings. They are also observed in the ${ }^{2} \mathrm{H}[40,41]$ and ${ }^{18} \mathrm{O}[39,42,43]$ isotopomers of gaseous TRN, and in simple chemical derivatives [44-57]. The numerous van der Waals complexes of tropolone are not discussed in this chapter.

The rotation-contortion-vibration electronic states of the nonrigid TRN and MA molecules are classified according to the $G_{4}$ molecular symmetry group using the permutation-inversion operations $E, P, i$, and $P^{*}$ [58]. The $G_{4}$ group is iso-<smiles>[2H]/C=C(/C)C(C)=O</smiles>

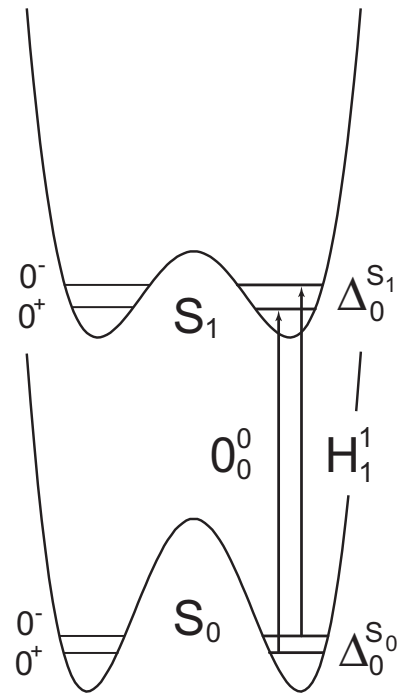<smiles>O=C1C=CC=CC=CC1O</smiles><smiles>O=CCO</smiles>

Figure 1.1 Symbolic representation of equal double-well potential energy functions for (A) tropolone and (B) malonaldehyde in the $S_{0}$ ground and lowest $S_{1}$ singlet electronic states. The spectral doublet of the $S_{1}-S_{0}$ zero-point transitions is shown. The doublet separation is $\left(\mathrm{H}_{1}{ }^{1}-0_{0}{ }^{0}\right)=\left|\Delta_{0} \mathrm{~S}_{1}-\Delta_{0} \mathrm{~S}_{0}\right|$. 
morphic to the $C_{2 \mathrm{v}}$ point group and the energy states are conventionally labeled using the $a_{1}, a_{2}, b_{1}$, and $b_{2}$ irreducible representations of $C_{2 v}$. The spectra observed for tropolone are complicated by many overlaps of the tunneling doubled fundamental, overtone, combination, and hot band vibrational transitions - the latter abundant at $25^{\circ} \mathrm{C}$. Many of the vibrational states are coupled by anharmonic resonance interactions affecting transition intensities more strongly than frequencies, and perturbing tunneling doublets [39].

The Schrödinger equation for a particle moving in a symmetrical one-dimensional (1D) double-well potential energy function (PEF) is readily solved for the lower energy levels and wavefunctions. The time-dependent wavefunctions produce probability density functions which coherently oscillate at a constant frequency between the two wells. In one's dreams multidimensional coordinate spaces of real molecules separate into simple 1D equations, and discovering the most effective real life coordinate separations and the most pertinent regions of the PES topography for complex molecules requires access to as much diverse and discriminating experimental information as possible. The status of research on coherent tunneling in the $\mathrm{S}_{0}$ and $\mathrm{S}_{1}$ electronic states of malonaldehyde and tropolone is discussed in Sections 1.2 and 1.3, respectively, with observations for several TRN derivatives sketched in Section 1.4, and a summary given in Section 1.5.

\section{2}

\section{Coherent Tunneling Splitting Phenomena in Malonaldehyde}

In 1976 Wilson's group published the first of several [9-12] studies on the microwave spectroscopy of the $\mathrm{C}, \mathrm{H}$, and $\mathrm{O}$ isotopomers of MA. These established its internally $\mathrm{H}$ bonded geometry, equal double-minimum nature of the PES, and coherent quantum tunneling activity. Their conclusions concerning the value of the zero-point (ZP) tunneling splitting, $\Delta_{0}$, from precise observations of tunneling-rotation transitions were supported by their early far-infrared spectral measurements in the $20 \mathrm{~cm}^{-1}$ region. Subsequent precision studies in this wavelength region by Firth et al. [17] and by Baba et al. [14] showed $\Delta_{0}=21.583 \mathrm{~cm}^{-1}$ for MA in its $\mathrm{S}_{0}$ ground electronic state. Baughcum et al. [10] reported $\Delta_{0}[\mathrm{MA}(\mathrm{OD})]=$ $2.915(4) \mathrm{cm}^{-1}$ for the symmetrical $\mathrm{MA}\left(\mathrm{D}_{6} \mathrm{D}_{7} \mathrm{D}_{9}\right)$ isotopomer (see atom numbering in Fig. 1.1), and slightly smaller values for nine other MA(OD) isotopomers, six of them incorporating ${ }^{13} \mathrm{C}$ or ${ }^{18} \mathrm{O}$ atoms.

ZP tunneling splitting values for MA in excited electronic states have proved elusive. Seliskar and Hoffman [20] reported vibronic spectral structure for the weak $\tilde{\mathrm{A}}{ }^{1} \mathrm{~B}_{1} \leftarrow \tilde{\mathrm{X}}{ }^{1} \mathrm{~A}_{1}\left(\pi^{*}-\mathrm{n}\right)\left[\mathrm{S}_{1} \leftarrow \mathrm{S}_{0}\right]$ absorption near $354 \mathrm{~nm}$ to have a spectral doublet separation (DS) of $7 \mathrm{~cm}^{-1}$. Arias et al. [21] applied the absorbance-based degenerate four-wave mixing (DFWM) spectroscopic method to the $\mathrm{S}_{1} \leftarrow \mathrm{S}_{0}$ transition. With higher intrinsic spectral resolution, and spectral clarification arising through the reduction of signal due to hot band transitions that is inherent in this nonlinear spectroscopic technique, they found DS $=\left|\Delta_{0} \mathrm{~S}_{1}-\Delta_{0} \mathrm{~S}_{0}\right| \sim 19 \mathrm{~cm}^{-1}$, to place the $\widetilde{\mathrm{A}}{ }^{1} \mathrm{~B}_{1}$ zero-point tunneling splitting value at $\Delta_{0} \mathrm{~S}_{1} \sim 2.5 \mathrm{~cm}^{-1}$. The $19 \mathrm{~cm}^{-1}$ 
DS value appears for the members of a $185 \mathrm{~cm}^{-1}$ vibronic progression, but details of the doublings for other vibrational states have not yet been reported. The strong quenching of $\Delta_{0} \mathrm{~S}_{1}$ relative to $\Delta_{0} \mathrm{~S}_{0}$ suggests the $\pi^{*} \leftarrow \mathrm{n}$ promotion increases the energy maximum and/or width of the effective PEF barrier [21].

Infrared spectra of gaseous MA and its deuterium isotopomers were reported at medium resolution by Seliskar and Hoffman [16] and by Smith et al. [15] and an overview at $1 \mathrm{~cm}^{-1}$ resolution is provided by Duan and Luckhaus [19]. At these modest resolutions the observed transition profiles include isolated PQR rotational envelopes as well as $\mathrm{Q}$ branch regions with multiple peaks suggesting doublets and probable hot band structures. Figure 1.2 is taken from Smith et al. [15] and shows peaks resolved in the $200-300 \mathrm{~cm}^{-1}$ region. Peaks \#20 and \#26 were assigned as representing the $v_{15}$ (nominal $\mathrm{O} \cdots \mathrm{O}$ stretch) and $v_{21}$ (out-ofplane skeletal deformation) fundamentals, respectively $[15,59,60]$, with no specific tunneling doublet or hot band assignments made. No evidence for the presence of resolved spectral tunneling doublets was reported by Firth et al. [17] for Ar-isolated MA or by Chiavassa et al. [18] in their IR studies of MA isotopomers isolated

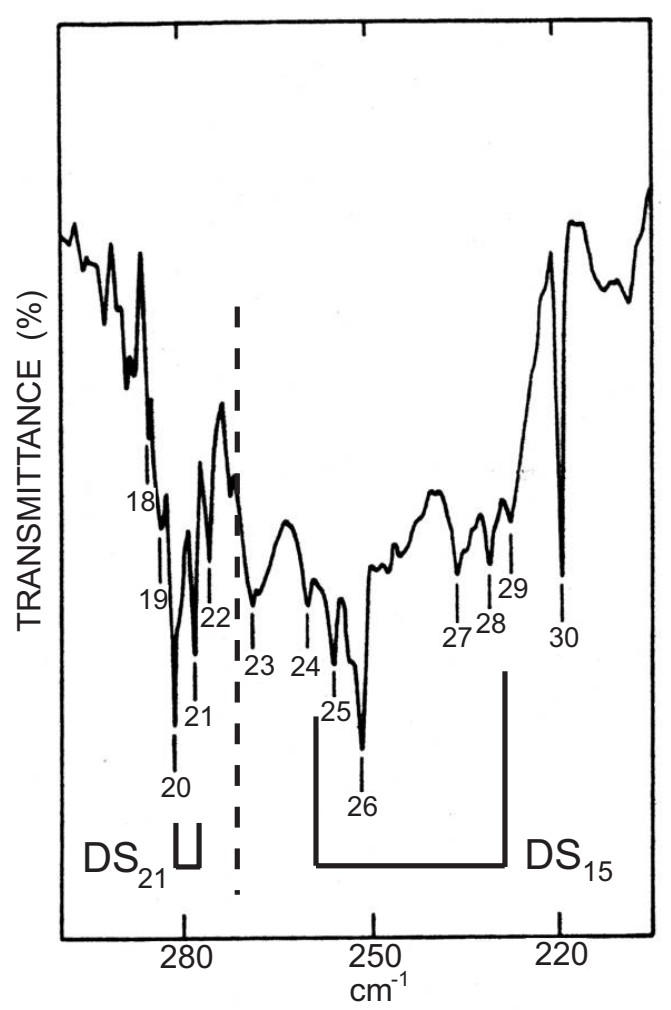

Figure 1.2 Infrared spectrum of $\mathrm{MA}(\mathrm{OH})$ in the $200-300 \mathrm{~cm}^{-1}$ region due to Smith et al. [15]. Spectral doublet separations $\mathrm{DS}_{21}$ and $\mathrm{DS}_{15}$ replace the single-peak assignments $v_{15}=$ peak 20 and $v_{21}=$ peak 26 . 
in Ar, Kr, and Xe matrices. Smith et al. [15] proposed a vibrational assignment for MA on the basis of their IR spectra for $\mathrm{H}$ and D isotopomers, comparative spectra for other substances, and a valence force field. Tayyari and Milani-Nejad [59] reassigned many fundamentals with the guidance of MP2/6-31G*//MP2/6-31G*(sp and $\mathrm{p}$ on $\mathrm{H})$ computations performed on $\mathrm{MA}, \mathrm{MA}\left(\mathrm{D}_{6} \mathrm{D}_{8}\right)$, and $\mathrm{MA}\left(\mathrm{D}_{7} \mathrm{D}_{9}\right)$. Alparone and Millefiori [60] affirmed the assignment through vibrational self-consistent field (VSCF) and correlation-corrected-VSCF computations introducing anharmonicities to estimate the fundamental isotopomer spectra.

Duan and Luckhaus [19] made a major experimental advance by using a Pb-salt diode laser to measure rotational transitions for $v_{6}$ in jet-cooled samples prepared by seeding MA into He carrier gas with expansion through a pulsed slit nozzle. The clarified spectrum allowed the origin for the $v_{6}{ }^{+} \leftarrow v_{0}{ }^{+}$(type A) tunneling doublet component to be evaluated as $1594.08983(26) \mathrm{cm}^{-1}$. Further, the determination of 12 rotation and centrifugal distortion constants provides comparisons for corresponding ZP state constants determined by Baba et al. [14]. The analysis of weak IR transitions produced a selection of parameters for the $v_{6}{ }^{-} \leftarrow v_{0}{ }^{-}$tunneling doublet component, to yield the remarkably small DS $=\Delta_{6}-\Delta_{0}=-0.03 \mathrm{~cm}^{-1}$ value for the $v_{6}$ fundamental $(\nu \mathrm{C}=\mathrm{O}+$ in-phase (iph) $\nu \mathrm{C}=\mathrm{C}+\delta \mathrm{COH}[60])$. Duan and Luckhaus suggested the tunneling enhancing effect of symmetrical $\mathrm{C}=\mathrm{C}-$ $\mathrm{C}=\mathrm{O}$ motion may be cancelled by the tunneling damping effect of concomitant $\mathrm{COH}$ angle widening, and they noted the challenge to theoretical treatments of the tunneling dynamics in MA provided by this accurate new experimental result.

While Smith et al. [15] eschewed the assignment of tunneling doublets, Seliskar and Hoffman [16] noted the $\mathrm{Q}$ branch doublet separations implying $\mathrm{DS}_{20}=$ $6.3 \mathrm{~cm}^{-1}$ for $v_{20}$ at $384 \mathrm{~cm}^{-1}$ (out-of-plane ring deformation) and $\mathrm{DS}_{14}=5.8 \mathrm{~cm}^{-1}$ for $v_{14}$ at $512 \mathrm{~cm}^{-1}$ (in-plane ring deformation). These $\mathrm{DS}_{\mathrm{V}}=\left|\Delta_{V}-\Delta_{0}\right|$ values suggest the upper state tunneling splittings $\Delta_{20}=15.3$ or $27.9 \mathrm{~cm}^{-1}$ and $\Delta_{14}=15.8$ or $27.4 \mathrm{~cm}^{-1}$. At $1 \mathrm{~cm}^{-1}$ resolution [19] there is a single sharp Q peak for $v_{6}$, and a single sharp Q peak for $v_{19}=766 \mathrm{~cm}^{-1}$. Isolated PQR envelopes with sharp Q spikes [19] reasonably imply $\Delta_{V} \sim \Delta_{0}$ for $v_{10}$ at $1260 \mathrm{~cm}^{-1}, v_{8}$ at $1346 \mathrm{~cm}^{-1}$, and $v_{7}$ at $1452 \mathrm{~cm}^{-1}$. To extract $\Delta_{V}$ values from Fig. 1.2 the cluster of peaks to the blue of $\sim 270 \mathrm{~cm}^{-1}$ (dotted line) is observed to be qualitatively similar to peaks shown for $v_{20}$ in the $370-410 \mathrm{~cm}^{-1}$ region (Fig. 7 of Ref. [15]). Assigning peak \#20= $282.2 \mathrm{~cm}^{-1}$ and $\# 21=279.3 \mathrm{~cm}^{-1}$ as the sharp Q spikes of type $\mathrm{C}$ absorption profiles for the doublet components of the out-of-plane - i.e. $v_{21}$ instead of $v_{15}$-vibration yields $\mathrm{DS}_{21}=\left|\Delta_{21}-\Delta_{0}\right|=2.9 \mathrm{~cm}^{-1}, \Delta_{21}=18.7$ or $24.5 \mathrm{~cm}^{-1}$, and $v_{21}=$ $1 / 2(282.2+279.3)=280.8 \mathrm{~cm}^{-1}$. The value $v_{21}=277.5 \mathrm{~cm}^{-1}$ is computed from the CC-VSCF treatment of Alparone and Millefiori [60] and the calculated minus observed value difference of $-1.2 \%$ is similar to the $-1.6 \%$ value found for $v_{20}$.

The cluster of peaks in Fig. 1.2 to the red of $\sim 270 \mathrm{~cm}^{-1}$ divide into similar groups with peaks \#23 to \#26, separated by $17.1 \mathrm{~cm}^{-1}$, and peaks \#27 to \#30, separated by $16.6 \mathrm{~cm}^{-1}$, spanning the high and low frequency groups, respectively. One of the two central peaks in each group is probably due to a type A Q spike of the Y polarized transition dipole, and the other probably to a hot band Q spike. The outer peaks are attributed to apparent band heads in the P and $\mathrm{R}$ branches. 
Pending further data, the doublet component origins are represented using peaks $\# 24$ and \#25 as $1 / 2(260.7+256.8)=258.8 \mathrm{~cm}^{-1}$ and peaks \#28 and \#29 as $1 / 2(231.9+228.5)=230.2 \mathrm{~cm}^{-1}$. Then $\mathrm{DS}_{15}=\left|\Delta_{15}-\Delta_{0}\right|=28.6 \mathrm{~cm}^{-1}$ yielding $\Delta_{15}=$ 7.0 or $50.2 \mathrm{~cm}^{-1}$, and $v_{15}=244.5 \mathrm{~cm}^{-1}$. The CC-VSCF computed estimate [60] for $v_{15}$ is $267.2 \mathrm{~cm}^{-1}$, a value $9.3 \%$ higher than $v_{15}=244.5 \mathrm{~cm}^{-1}$. For the original assignment the differences are $277.5-252=25.5(10.1 \%)$ and $267.2-282=$ $-14.8 \mathrm{~cm}^{-1}(-5.2 \%)$.

The experimentally based $\Delta_{V}$ values for nine fundamentals are listed in column 3 of Table 1.1 and approximate descriptions of the vibrations by Alparone and Millefiori [60], whose computations fit the observed IR spectrum to 2 or $3 \%$, are listed in the right-most column. In column 4 the $\Delta_{V} / \Delta_{0}$ ratios are listed next to theoretical ratios [61-64] discussed below. Of the nine experimental values only the four lower frequency skeletal displacements - two in-plane and two out-ofplane - appear to significantly impact the tunneling process. Experimental splittings remain unknown for the vibrations dominated by motion of the tunneling $\mathrm{H}$ (that is the $v \mathrm{OH}, \delta \mathrm{COH}$, and $\gamma \mathrm{OH}$ internal coordinates). The $v_{\mathrm{OH}}$ stretching vibration is anharmonically coupled to many nearby overtone and combination vibrations and its doublet separation is concealed in the broad IR absorption profile. The vibrational compositions in Table 1.1 show that the fundamentals containing the $\gamma \mathrm{OH}$ torsion and $\delta \mathrm{COH}$ bending coordinates are strongly mixed with other internal coordinates.

Carrington and Miller [65] using 2D and Shida et al. [66] using 3D quantum chemical reaction surface models produced the first of many extant theoretical estimates for $\Delta_{0}$ and vibrational state specific splittings $\Delta_{V}$ for MA. The differences of $2 \mathrm{D}$ and $3 \mathrm{D}$ computations are very noticeable and they are the exclusive topic of a recent article by Yagi et al. [67]. The theoretical approaches to MA vary from extensions of the cited early work, to that on full multidimensional instanton computations by Smedarchina et al. [68], to work on new techniques such as the short time propagation method being tested by Giese and Kühn [69]. Došlic and Kühn [70] studied modes in the OHO fragment in 4D computation. Tautermann et al. [71] developed an improved semiclassical method for determining the optimized multidimensional tunneling path between the straight line and minimum energy path routes. The ZP corrected PES was constructed using low-level energy points calculated using B3LYP/6-31G(d) density functional theory (DFT) methodology and this PES was then scaled to match a few high level G3(MP2) reference points. The procedure produced computed $\Delta_{0}$ values for $(\mathrm{HF})_{2}, \mathrm{MA}$, and TRN that were each in good agreement with experiment.

PES features are now routinely computed using quantum chemistry methods that must necessarily include estimates for the electron correlation energy. The computed SP energy values depend strongly on the level of theory used, as exemplified by the range $10.34 \mathrm{kcal} \mathrm{mol}^{-1}$ (RHF/6-31G***) to $2.86 \mathrm{kcal} / \mathrm{mol}$ [B3LYP/6$311+\mathrm{G}(2 \mathrm{~d}, \mathrm{p})]$ reported by Benderskii et al. [61]. Barone and Adamo [72] proposed $4.3 \mathrm{kcal} \mathrm{mol}^{-1}$ as the best value for the SP barrier for MA on the basis of high level comparative studies. Sadhukhan et al. [73] reported $3.9 \mathrm{kcal} \mathrm{mol}^{-1}$ for $\operatorname{CCSD}(\mathrm{T}) /$ cc-pVDZ computation. 
Yagi et al. [74] determined an ab initio PES for MA, based on 698 reference points in the full 21D vibrational space, in work made possible through the use of a modified Shepard interpolation method. For this PES they estimated $\Delta_{0}=13.9 \mathrm{~cm}^{-1}$ by using the semiclassical trajectory method proposed by Makri and Miller [75]. The $\Delta_{0}$ value can also be calculated using exact quantum dynamics methods, and Coutinho-Neto et al. [76] computed $\Delta_{0}$ using the diffusion Monte Carlo based projection operator imaginary time spectral evolution method to obtain $\Delta_{0}=25.7 \pm 0.3 \mathrm{~cm}^{-1}$. The multiconfigurational time-dependent Hartree approach yielded $\Delta_{0}=25 \mathrm{~cm}^{-1}$ to about $10 \%$ accuracy. These results establish an independent benchmark comparison for the various semiclassical results. Mil'nikov and Nakamura [77] reformulated instanton theory to allow "practical" computations of multidimensional tunneling splittings which, with some refinements, was applied [78] to the 21D PES to obtain $\Delta_{0}=30.7 \mathrm{~cm}^{-1}$.

The reformulated instanton theory introduces a variational procedure to minimize the classical action functional determining the instanton trajectory. Few iterations of the procedure are required to converge an initial approximate trajectory into the instanton. Because the required quantum chemical PES computations are focused on the instanton region of the PES, relatively few points (energy, gradient, Hessian) are required. The method allows the instanton obtained from a low level PES to be the starting point for determining the instanton generated from a higher level PES [77, 78]. Mil'nikov et al. [78] could address MA - with full 21D consideration - using ab initio PESs obtained at high levels of quantum chemistry. The new theoretical development produces smooth instanton paths (unlike results found for the PES obtained through the Shepard interpolation procedure). The reduced quantum chemistry work load allowed the variational instanton method to determine $\Delta_{0}=16.4 \mathrm{~cm}^{-1}$ for MA at the $\operatorname{CCSD}(\mathrm{T}) /$ (aug-)pVDZ level of PES computation. With recognition that full computation using the (aug-)cc-pVTZ basis would require more than 5 years of CPU time because of the Hessian calculation, a partial computation with the instanton preexponential $B$ and $S_{1}$ factors transferred from MP2/cc-pVDZ computations yielded $\Delta_{0}=21.2 \mathrm{~cm}^{-1}$ - very near the $21.6 \mathrm{~cm}^{-1}$ experimental result. Similarly, $\Delta_{0}[\mathrm{MA}(\mathrm{OD})]=3.0 \mathrm{~cm}^{-1}$ was calculated with $2.9 \mathrm{~cm}^{-1}$ observed. Mil'nikhov et al. [78] estimate the error in the computed $\Delta_{0}$ value for MA to be at most $10 \%$. This work re-emphasizes the sensitivity of the $\Delta_{0}$ computation to the PES determination, while taking advantage of the lower sensitivity of the $B$ and $S_{1}$ pre-exponential factor computations to the quantum chemical method. The developmental work [77] included the demonstration that the utilized multidimensional path integral method and the multidimensional Wentzel-Kramers-Brillouin (WKB) method yield equivalent results. The variational instanton method has not yet been applied to the tunneling splittings of vibrational excited states of MA.

Benderskii et al. [61] developed a theory called the perturbative instanton approach (PIA) which they applied to MA in full 21D treatment. An important feature of their work is the expression of the carefully partitioned and symmetrized Hamiltonian in expansions using dimensionless variables including a semiclassical parameter called $\gamma$. The $\gamma$ value allows scaling of the tunneling 


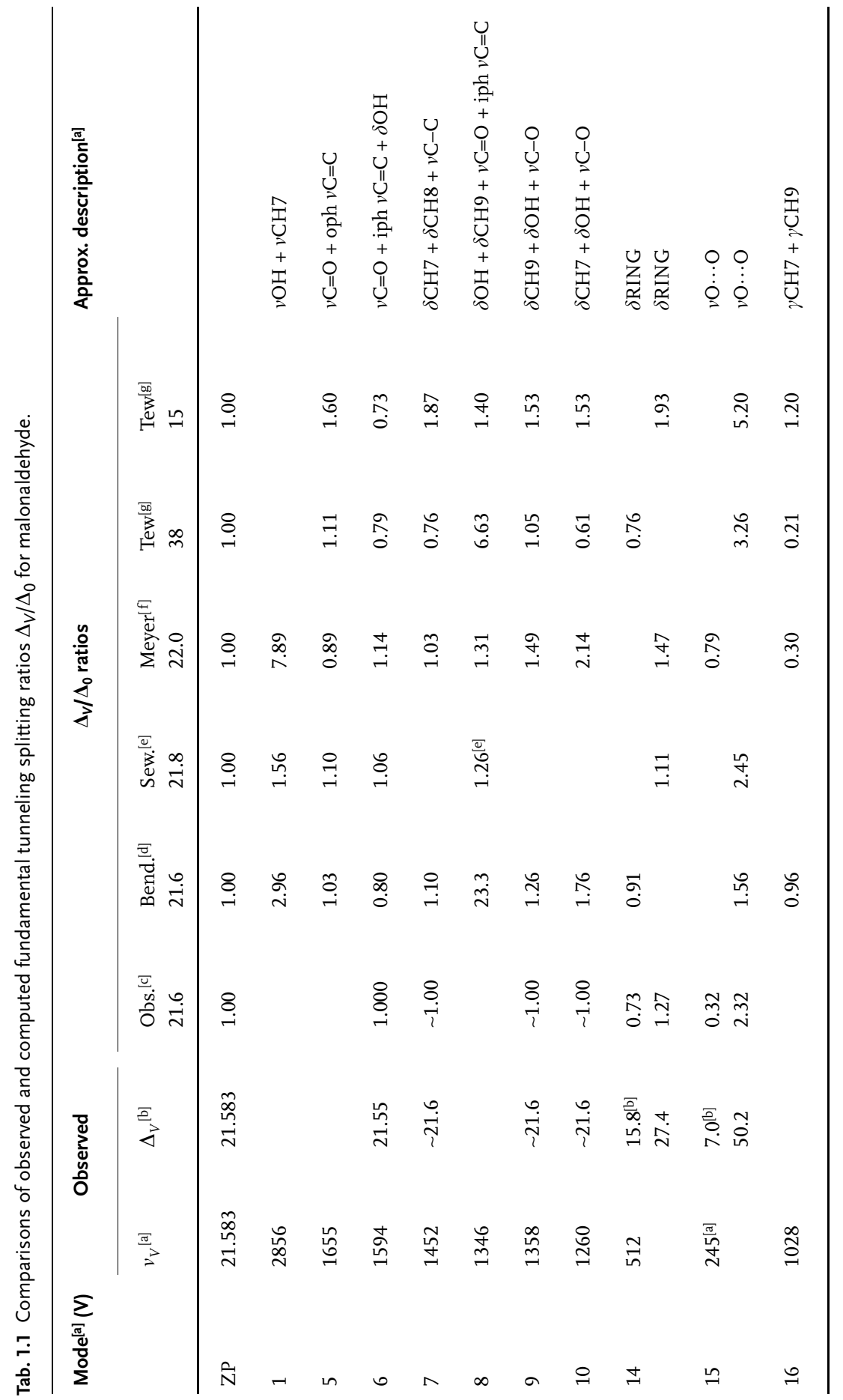




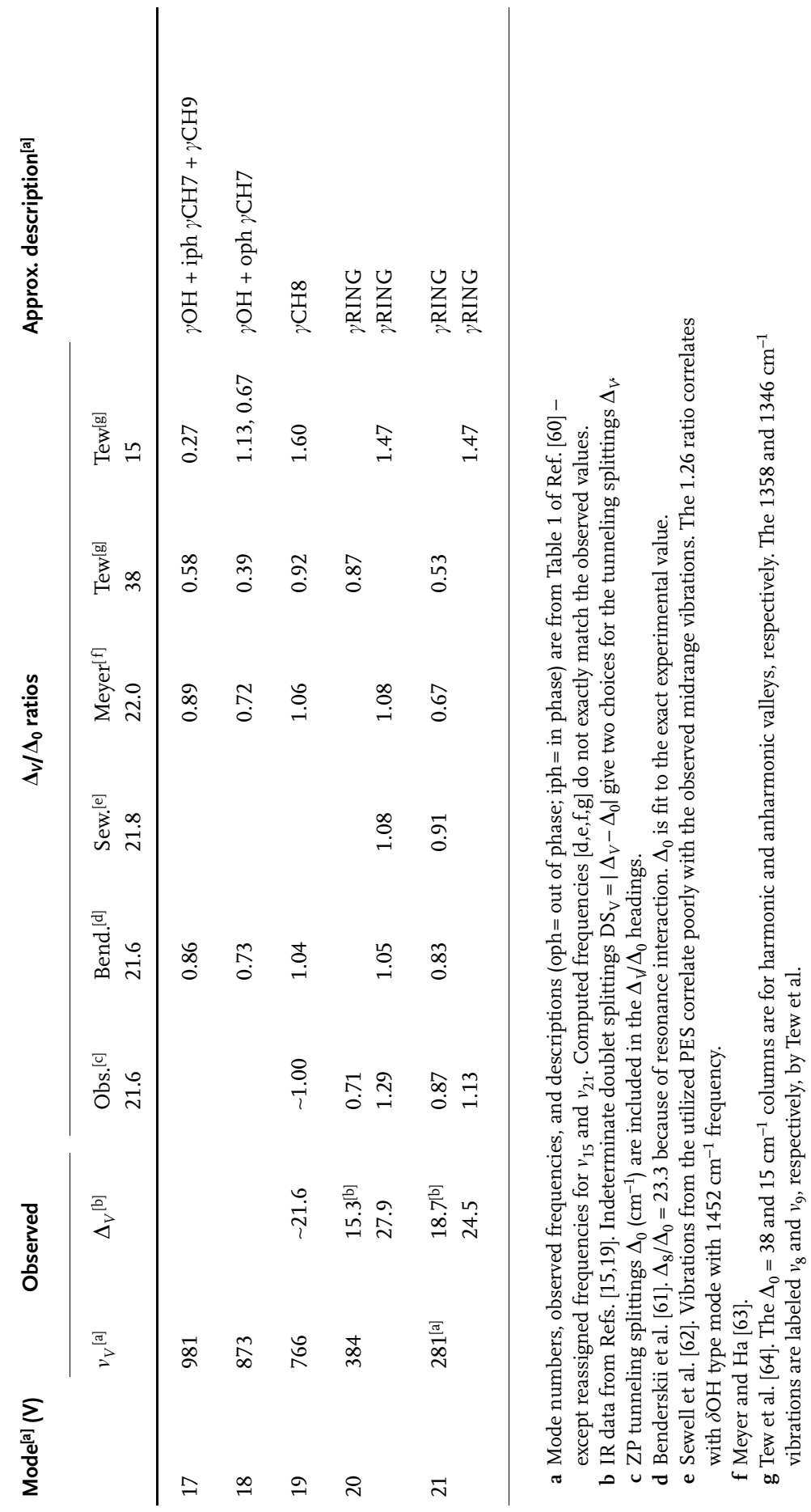


splitting $\Delta_{0}$ and SP barrier height, which are quite sensitive to $\gamma$, as well as spectral densities and other quantities that are relatively insensitive to $\gamma$. The selected PES was scaled with $\gamma$ chosen to duplicate the experimental $\Delta_{0}=21.6 \mathrm{~cm}^{-1}$ splitting. The resulting SP energy is $4.30 \mathrm{kcal} \mathrm{mol}^{-1}$ - the same value chosen by Barone and Adamo [72] as the best value for MA on the basis of their comparative quantum chemical computations. Conversely, choosing $\gamma$ to reproduce this ab initio SP energy value yields the observed $\Delta_{0}$ splitting. The full 21D vibrational spectrum and $21 \Delta_{V}$ values were computed in the PIA. In column 5 of Table 1.1 a selection of the resulting $\Delta_{V} \Delta_{0}$ ratios are listed for comparison with columns containing the available experimental values and results from other computational procedures. At this stage, the appreciable dispersion of the computed PES topologies, vibrational spectra, and tunneling splitting values favors comparison of the results through ratios. Benderskii et al. [61] present a detailed description of the dynamics calculated for MA and several isotopomers. They refer to $v_{8}$, observed at $1346 \mathrm{~cm}^{-1}$, as the tunneling coordinate with $v_{1}\left(\mathrm{OH}\right.$ stretching) and $v_{15}(\mathrm{O} \cdots \mathrm{O}$ stretching) designated as promoting modes. The very large $\left(500 \mathrm{~cm}^{-1}\right)$ computed splitting obtained for $v_{8}$ is attributed to a resonance interaction with $v_{1}$ - also stated to be resonantly coupled with two nearby $\mathrm{CH}$ stretching modes with computed tunneling splittings above $100 \mathrm{~cm}^{-1}$. The values $\Delta_{8} / \Delta_{0}=23.3, \Delta_{3} / \Delta_{0}=7.55$, and $\Delta_{2} / \Delta_{0}=$ 5.89 are by far the largest ratios arising in this study.

Column 6 of Table 1.1 presents several $\Delta_{V} / \Delta_{0}$ ratios obtained from the full 21D classical trajectory/WKB computations of Sewell et al. [62]. The utilized PES is based on the 1983 valence force field, and early vibrational assignments of Smith et al. [15] - some of which differ from later work [59, 60]. Many of the computed splitting ratios are therefore not entered in Table 1.1. The PES was given Morse functions for the bond stretching coordinates. At $10.0 \mathrm{kcal} \mathrm{mol}^{-1}$ the SP energy is very high but the barrier is noticeably narrow in its upper reaches. The computed ZP tunneling splitting is $\Delta_{0}=21.8 \mathrm{~cm}^{-1}$, fortuitously close to the $21.6 \mathrm{~cm}^{-1}$ observed value. The $\Delta_{V} / \Delta_{0}$ ratios listed in column 6 are those with the closest agreement to current understanding of the vibrational structure and, for whatever reason, they follow the lead of $\Delta_{0}$ by showing quite good agreement with the experimental results.

Full 21D computations were produced using direct quantum chemical methods by Meyer and Ha [63] and by Tew et al. [64, 79]. Meyer and Ha developed a reference reaction path model applied using a PES with the SP barrier of $3.27 \mathrm{kcal} \mathrm{mol}^{-1}$. They obtained $\Delta_{0}=22.0 \mathrm{~cm}^{-1}$ for $\mathrm{MA}(\mathrm{OH}), 3.8 \mathrm{~cm}^{-1}$ for MA(OD), and values for all $21 \Delta_{V}$ splittings. Some of the resulting $\Delta_{V} / \Delta_{0}$ ratios are listed in column 7 of Table 1.1, where it is seen that a large splitting is predicted for $v_{1}(\mathrm{OH}$ stretching) and that damping of the ZP splitting is predicted for the low frequency $\mathrm{H}$ transfer coordinate $v_{15}\left(\mathrm{O} \cdots \mathrm{O}\right.$ stretching). The largest ratios are $\Delta_{1} / \Delta_{0}=7.89$, $\Delta_{10} / \Delta_{0}=2.14$, and $\Delta_{13} / \Delta_{0}=1.66 . \Delta_{V}$ values for several modes are significantly larger than suggested by the experimental data. Tew et al. developed an internal coordinate reaction path Hamiltonian allowing the usual division of coordinates: a single large amplitude motion with 20 orthogonal normal modes. In their work the energy levels are solved by matrix diagonalization (that is, by variational con- 
figuration interaction computations that were performed on three increasingly inclusive approximations for the Hamiltonian.) They initially [79] applied the method to MA using a PES determined by DFT. This work was followed [64] by an article concentrating on the use of PESs determined using the MP2 approximation for electron correlation energies because of convergence inadequacies arising through the use of DFT. In columns 8 and 9 of Table 1.1 the listing of computed $\Delta_{V} / \Delta_{0}$ ratios is obtained from results for the highest level Hamiltonians using a computed harmonic PES valley $\left(\Delta_{0}=38 \mathrm{~cm}^{-1}\right.$, column 8) and a computed anharmonic PES valley $\left(\Delta_{0}=15 \mathrm{~cm}^{-1}\right.$, column 9$)$. It is noted that the computations for the harmonic valley predict quenching of the ZP tunneling on exciting $v_{20}$ or $v_{21}$, while for the anharmonic valley the computations predict enhancement instead. For the harmonic valley the three largest ratios are $\Delta_{8} / \Delta_{0}=6.63, \Delta_{15} / \Delta_{0}=3.26$, and $\Delta_{13} / \Delta_{0}=2.82$. For the anharmonic valley they are $\Delta_{15} / \Delta_{0}=5.20, \Delta_{13} / \Delta_{0}=$ 2.00 , and $\Delta_{14} / \Delta_{0}=1.93$. Good convergence was obtained for the lower vibrational frequencies but converged $\Delta_{V}$ splittings could not be obtained for the $\mathrm{OH} / \mathrm{CH}$ stretching frequencies.

\section{3}

\section{Coherent Tunneling Phenomena in Tropolone}

The first experimental evidence for coherent tunneling in the moderately large molecules of interest in this chapter was reported for TRN in 1972 by Alves and Hollas [22]. Vibronic absorption bands of gaseous TRN $(\mathrm{OH})$ and TRN(OD) in the $370 \mathrm{~nm}$ region were recorded at relatively high spectral resolution to clearly show rovibronic fine structures with peak spacings of $\sim 0.1 \mathrm{~cm}^{-1}$. Observed band head peaks were systematically paired as spectral doublets [22, 23] with separations both larger and smaller than the value of $18.93 \mathrm{~cm}^{-1}$ determined for the ZP origin doublet. This important work on gaseous tropolone showed that readily measured coherent tunneling processes occur over at least the lower $700 \mathrm{~cm}^{-1}$ range of vibrational excited states in the $\widetilde{A}{ }^{1} B_{2}\left(S_{1}\right)$ electronic state and it stimulated many experimental and theoretical studies on this system.

For TRN $(\mathrm{OH})$ Alves and Hollas assigned the ZP doublet transitions labeled $0_{0}{ }^{0}$ $\left(0^{+}-0^{+}\right)$and $\mathrm{H}_{1}{ }^{1}\left(0^{-}-0^{-}\right)$in Fig. 1.3 and determined their origins to be 27017.54 and $27036.47 \mathrm{~cm}^{-1}$, respectively. The spectral doublet separation is DS $=\mid \Delta_{0} \mathrm{~S}_{1}$ $\Delta_{0} \mathrm{~S}_{0} \mid=18.93 \mathrm{~cm}^{-1}$; for TRN(OD) they found DS $=2.2 \mathrm{~cm}^{-1}$. Independent data are required to determine the individual $\Delta_{0} \mathrm{~S}_{1}$ and $\Delta_{0} \mathrm{~S}_{0}$ values, and Alves and Hollas were limited to recognizing that $\Delta_{0} \mathrm{~S}_{1}$ is $18.93 \mathrm{~cm}^{-1}$ larger than $\Delta_{0} \mathrm{~S}_{0}$. In 1999 Tanaka et al. [32] used microwave spectroscopy to determine that (rounded to three digits) $\Delta_{0} \mathrm{~S}_{0}=0.974 \mathrm{~cm}^{-1}$ for gaseous tropolone. The untruncated ZP splitting, $\Delta_{0} \mathrm{~S}_{0}=29193.788(26) \mathrm{MHz}$, was determined using a single interaction parameter with six rotational and centrifugal distortion constants each for the symmetric $\left(0^{+}\right)$and antisymmetric $\left(0^{-}\right)$tunneling states. Stark effect measurements on the $3_{2,1} \leftarrow 2_{2,0}$ and $3_{2,2} \leftarrow 2_{2,1}$ rotational transitions show the average dipole moments of gaseous tropolone along the $Z$ (longitudinal) axis in these rotational 


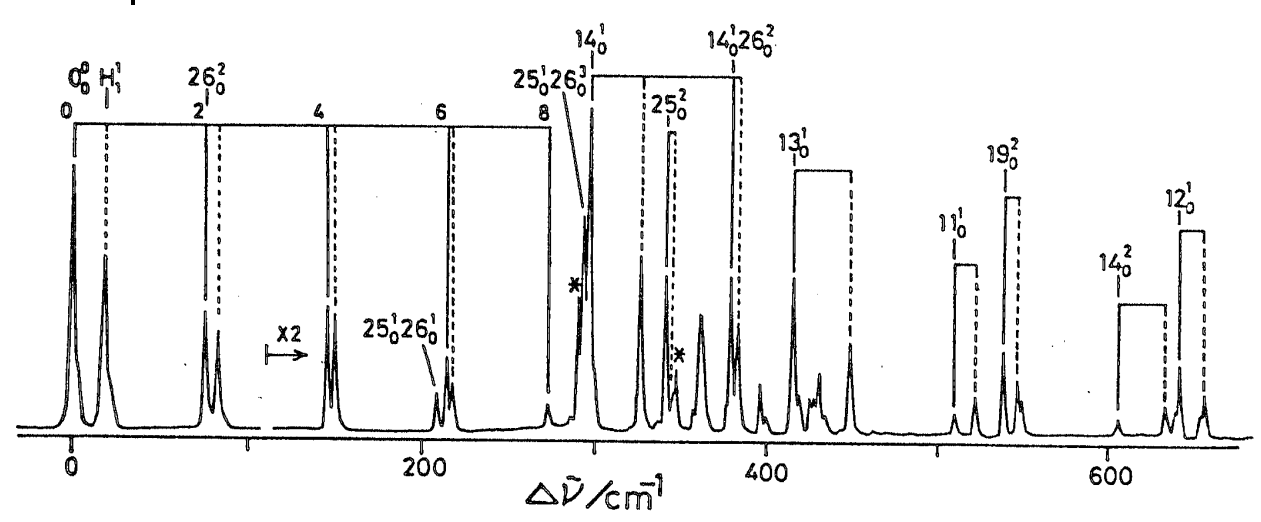

Figure 1.3 Vibrational state-specific doublets observed in the $\mathrm{S}_{1}-\mathrm{S}_{0}$ fluorescence excitation spectrum of jet-cooled $\operatorname{TRN}(\mathrm{OH})$ by Sekiya et al. [31].

states are 3.428(50) $\mathrm{D}$ for $0^{+}$and 3.438(50) $\mathrm{D}$ for $0^{-}$. The analogous dipole moment component for MA is $2.59 \mathrm{D}$ [10]. The microwave study verified beyond any doubt that tropolone possesses a symmetrical double-minimum PES in its $\mathrm{S}_{0}$ electronic state, that the molecule undergoes coherent state-specific rotation-tunneling transitions, and that there are small but measurable differences between rotational constants of the $0^{+}$and $0^{-}$states. The node in the $0^{-}$tunneling wavefunction may cause the inequality $\mathrm{A}^{+}=2743.0931(54)>\mathrm{A}^{-}=2742.7181(114) \mathrm{MHz}$ [32].

A few years after Alves and Hollas studied the vibronic absorption spectrum of gaseous tropolone in a $1 \mathrm{~m}$ cell at room temperature, the application of pulsed UV laser spectroscopy to gaseous samples cooled in pulsed molecular beams became possible. Passing the carrier gas over tropolone crystals loaded into a pulsed nozzle at $45^{\circ} \mathrm{C}$ produces excellent samples with rotational temperatures $\sim 3 \mathrm{~K}$ and vibrational temperatures $\sim 15 \mathrm{~K}$. The initial works were by Tomioka et al. [26], Redington et al. [28], and Sekiya et al. [30]. With the elimination of hot bands and with rotational envelopes reduced to widths of about $2 \mathrm{~cm}^{-1}[28,29]$, the $\mathrm{S}_{1}-\mathrm{S}_{0}$ fluorescence excitation spectrum is greatly simplified compared with the $25^{\circ} \mathrm{C}$ absorption spectrum. In the best resolved fluorescence excitation data the ZP origin doublet components show peaks with central dips which, taken as the band origins, yield DS $=18.90 \mathrm{~cm}^{-1}$, in good agreement with the value reported by Alves and Hollas.

The most recent publications on the $\mathrm{S}_{1}-\mathrm{S}_{0}$ transition of TRN $(\mathrm{OH})$ are by Bracamonte and Vacarro [37, 38], who applied their polarization resolved DFWM spectroscopy method [37] to TRN vapor at its room temperature sublimation pressure. This remarkable nonlinear spectroscopic method simplifies the spectrum by allowing $\mathrm{Q}$ branch $(\Delta J=0)$ transitions to be observed with greatly diminished $\mathrm{R}$ and $P$ branch $(\Delta J= \pm 1)$ signal and, with rotation of a polarization element, vice versa. This selective nonlinear optical technique also produces a large reduction in the signal generated by hot bands, as noted in the above discussion for MA. How- 
ever, with optical saturation the hot band Q branch signals are sufficiently strong that some of their band origins could be approximated [38]. With the $\mathrm{S}_{0}$ ground state rotational parameters known from the work of Tanaka et al. [32], the $\mathrm{S}_{1}$ state parameters, including the coherent tunneling splitting value $\Delta_{0} \mathrm{~S}_{1}=19.846(25) \mathrm{cm}^{-1}$, were accurately determined by Bracamante and Vaccaro [38]. The extra large inertial defects $\Delta I_{0}+\mathrm{S}_{1}=-0.802(86)$ and $\Delta I_{0}-\mathrm{S}_{1}=-0.882(89)$ amu $\AA^{2}$ led them to conclude that the geometry of tropolone in the $\mathrm{S}_{1}$ electronic state is slightly nonplanar.

Tropolone is easily deuterated and the $\mathrm{S}_{1}-\mathrm{S}_{0}$ fluorescence excitation spectrum of jet-cooled TRN(OD) was reported by Sekiya et al. [40]. The observed doublet separation, $\mathrm{DS}=2 \mathrm{~cm}^{-1}$, is near the $2.2 \mathrm{~cm}^{-1}$ value reported by Alves and Hollis [22], and about $10 \%$ of the value observed for $\mathrm{TRN}(\mathrm{OH})$. Presently the only available experimental estimate for the $\mathrm{ZP}$ tunneling splitting of $\mathrm{S}_{0} \mathrm{TRN}(\mathrm{OD})$ is $\Delta_{0} \mathrm{~S}_{0} \leq 0.17 \mathrm{~cm}^{-1}$ [80] obtained using a chloroform solvent and NMR spectroscopy to measure the deuteron spin-lattice relaxation time.

The $\mathrm{S}_{1}-\mathrm{S}_{0}$ fluorescence excitation and dispersed fluorescence spectra of TRN $(\mathrm{OH})$ and TRN(OD) isolated in Ne matrices at $\sim 3.5 \mathrm{~K}$ were studied by Rossetti and Brus [25] using UV pulsed laser excitation. Low temperature Ne matrixisolation sampling is advantageous because, in principle, the sample monomers are dispersed in identical trapping sites resulting in a single sharp peak very near the gas-phase origin for each spectral transition. Rossetti and Brus found that the $\mathrm{S}_{1}-\mathrm{S}_{0}$ band origin transitions are doublets with the separations DS $=21 \pm 2 \mathrm{~cm}^{-1}$ for $\mathrm{TRN}(\mathrm{OH})$ and $\mathrm{DS}=7 \pm 1 \mathrm{~cm}^{-1}$ for $\mathrm{TRN}(\mathrm{OD})$. These values are larger than the gas phase values by $2 \pm 2 \mathrm{~cm}^{-1}$ for TRN $(\mathrm{OH})$ and $5 \pm 1 \mathrm{~cm}^{-1}$ for TRN(OD). According to theory [81], coupling a tunneling system to a bath of harmonic oscillators produces a damping of the tunneling splittings. The increased spectral doublet separations observed for Ne-isolated tropolone are explainable [82] through the interaction of nonplanar $\mathrm{S}_{1}$ tropolone with an asymmetric Ne matrix trapping site.

The large observed inertial defects [38], and molecular orbital (MO) computations reported by Wójcik et al. [83], suggest the geometry of tropolone is probably slightly nonplanar in the $\mathrm{S}_{1}$ state. For discussion it is assumed to be deformed towards a boat shape. (In the crystal state $\mathrm{S}_{0}$ tropolone is boat-shaped with folding angles of 1 or $2^{\circ}[84]$ ). Tautomerization inverts the concavity of the TRN geometry to, for example, uncradle an initially cradled $\mathrm{Ne}$ atom in the asymmetric trapping site. The small reorientation of the TRN position causes minimal disturbance of the trapping site configuration, but it produces an offset between energy minima of the two tautomer configurations to distinguish the behavior of planar $\mathrm{S}_{0}$ and slightly nonplanar $\mathrm{S}_{1}$ tropolone in an asymmetric Ne matrix trapping site.

Hameka and de la Vega [85] presented a theoretical description of the ZP energy levels and probability density functions for a particle in a slightly asymmetric double-well PEF. They analyzed the model behavior in terms of two basic parameters: $\delta$ (the energy offset between the two PEF minima), and $\Delta$ (the tunneling splitting that occurs for the limiting equal double-well PEF - namely at $\delta=0$ ). Equations presented by Hameka and de la Vega are easily combined [39, 82] into the equation $\left({ }^{\text {as }} \Delta\right)^{2}=\delta^{2}+\Delta^{2}$, where as $\Delta$ is the splitting of the ZP energy levels in the PEF. 
Except for the small nonplanarity of $\mathrm{S}_{1} \mathrm{TRN}$, the $\mathrm{S}_{0}$ and $\mathrm{S}_{1}$ geometries, Ne matrix trapping sites, and (probably) fundamental tautomerization processes of TRN $(\mathrm{OH})$ and TRN(OD) are similar. For gaseous TRN in the $\mathrm{S}_{0}$ and $\mathrm{S}_{1}$ states the PEF is symmetrical so that $\delta=0$ and $\Delta=\Delta_{0}={ }^{\text {as }} \Delta$. The observed IR and UV spectral data for Ne-isolated TRN suggest $\delta \sim 0$ for TRN in the $\mathrm{S}_{0}$ state, and $\delta=7 \pm 1 \mathrm{~cm}^{-1}$ for TRN in the $\mathrm{S}_{1}$ state. The latter value accounts for the comparative spectral doublet behavior of the $\mathrm{S}_{1}-\mathrm{S}_{0}$ origin bands of Ne-isolated and gaseous TRN $(\mathrm{OH})$ and TRN(OD). The spectral data also suggest that the Ne trapping site environment damps the $\Delta$ tunneling splitting values by an amount $d$ taken to be the same for the $\mathrm{S}_{0}$ and $\mathrm{S}_{1}$ states of both isotopomers (if $d>\Delta_{V}$, the $\Delta_{V}$ value is fully quenched). Dampings $1 \leq d \leq 2.5 \mathrm{~cm}^{-1}$ therefore completely quench the TRN $(\mathrm{OH})$ splitting $\Delta_{0} \mathrm{~S}_{0}=0.974 \mathrm{~cm}^{-1}$, but only mildly damp $\Delta_{0} \mathrm{~S}_{1}=19.864 \mathrm{~cm}^{-1}$. The value $d=2.5 \mathrm{~cm}^{-1}$ is the maximum value consistent with error bars of the observed spectral doublets.

The modeling computations of Makri and Miller [81] show that if a specific tunneling system is coupled to a high bath frequency the system tunneling splitting is only slightly damped, whereas if the coupling is to a low bath frequency the damping is strong. An analogous situation is to compare dampings generated by the couplings of a specific bath frequency to different tunneling systems, say high barrier system A with splitting $\Delta_{0}$ and low barrier system B with splitting $10 \Delta_{0}$. Compared to the bath oscillator the parameters of system A would be high [that is, relative to them the bath frequency would be low (say below the barrier) with strong damping of the splitting]. Similarly, the parameters of system B would be low [that is, relative to them the bath frequency would be high (say above the barrier) with weak damping of the splitting]. A specific Ne trapping site, with its fixed distribution of vibrational frequencies, can thus be envisioned as strongly damping (percentage wise) a tunneling system with a small $\Delta_{0}$ value $\left(\Delta_{0} \mathrm{~S}_{0}\right.$ of TRN), and weakly damping (percentage wise) a tunneling system with a large $\Delta_{0}$ value $\left(\Delta_{0} \mathrm{~S}_{1}\right.$ of TRN).

Fluorescence excitation [43] and IR absorption [39] spectra of 16,16 $\mathrm{TRN}(\mathrm{OH})$, $16,18 \mathrm{TRN}(\mathrm{OH})$, and ${ }^{18,18} \mathrm{TRN}(\mathrm{OH})$ show that an asymmetric double-well PES occurs for gaseous ${ }^{16,18} \mathrm{TRN}$ in the $\mathrm{S}_{0}$ and $\mathrm{S}_{1}$ electronic states. This is due to unequal anharmonic vibrational energy contributions to the effective PES at the limiting ${ }^{16} \mathrm{OH} \cdots{ }^{18} \mathrm{O}$ and ${ }^{16} \mathrm{O} \cdots \mathrm{H}^{18} \mathrm{O}$ tautomer configurations. The lowered symmetry of ${ }^{16,18} \mathrm{TRN}(\mathrm{OH})$ facilitates the observation of four (rather than two) fluorescence excitation transitions [43]. Combination differences of the observed transitions show the ZP levels of ${ }^{16,18} \mathrm{TRN}(\mathrm{OH})$ are separated by $1.7 \mathrm{~cm}^{-1}$ in the $\mathrm{S}_{0}$ state [compared to $\Delta_{0} \mathrm{~S}_{0}=0.974 \mathrm{~cm}^{-1}$ for ${ }^{16,16} \mathrm{TRN}(\mathrm{OH})$ ].

The fluorescence excitation spectra of jet-cooled TRN $(\mathrm{OH})$ and TRN(OD) show vibrational transitions in the $\mathrm{S}_{1}$ state reaching to about $700 \mathrm{~cm}^{-1}$. The lowest frequency fundamental of $\mathrm{TRN}(\mathrm{OH})$ occurs at $39 \mathrm{~cm}^{-1}$ in the $\mathrm{S}_{1}$ state, and at $110 \mathrm{~cm}^{-1}$ in the $\mathrm{S}_{0}$ state. The MO-computed normal coordinate for the $\mathrm{S}_{0}$ state suggests primary OCCO/skeletal twisting, rather than wagging, character. If its true nature is torsional, it should be identified as $v_{19}\left(\mathrm{a}_{2}\right)$ rather than as $v_{26}\left(\mathrm{~b}_{1}\right)$. The low frequency values reflect the competition between drives favoring planar 
geometry (the internal $\mathrm{H}$ bond and $\pi$ resonance interactions), and drives favoring nonplanar geometry (the relaxation of heptacyclic ring strain, and of cis OCCO alignment).

The fluorescence excitation spectrum of jet-cooled $\mathrm{TRN}(\mathrm{OH})$ as presented by Sekiya et al. [31] is shown in Fig. 1.3. A series of vibrational state-specific tunneling doublets (labeled $26_{0} \mathrm{~V}$ ) are seen to proceed to the blue of the $\mathrm{S}_{1}-\mathrm{S}_{0}$ origin doublet. These involve even numbered vibrational excitations of the $39 \mathrm{~cm}^{-1}$ mode in the $S_{1}$ state. The spectral doublet separations, $7.23,4.72,3.51, \cdots \mathrm{cm}^{-1}$, are strongly damped from the $18.90 \mathrm{~cm}^{-1}$ value for the origin band. The figure also shows vibrationally enhanced doublet separations for $v_{13}\left(414.66,446.7 \mathrm{~cm}^{-1}\right)$ and $v_{14}$ (295.82, $326.41 \mathrm{~cm}^{-1}$ ) [28-31], which are $\mathrm{a}_{1}$ skeletal deformation fundamentals with strong components of $\mathrm{O} \cdots \mathrm{O}$ stretching. The above vibrational state-specific doublet separations for the $S_{1}$ state are collected in column 3 Table 1.2, where they are compared with theoretical splittings computed by Wójcik et al. [83] as noted below.

Many of the spectral doublet separations observed for $\mathrm{S}_{1}$ state vibrations are large and easily measured and, in principle, dispersion of the fluorescence excited from the $\mathrm{S}_{1}$ states allows many $\mathrm{S}_{0}$ vibrational states to be probed. The individual doublet components of the $\mathrm{S}_{1}-\mathrm{S}_{0}$ origin or other sufficiently strongly fluorescent doublet component can be excited with laser pulses to generate fluorescence transitions of the same parity terminating on $\mathrm{S}_{0}$ vibrational states. Values for the frequencies of $v_{13}, v_{14}$, and other fundamental and excited state transitions are assigned [27, 31], but most $\mathrm{S}_{0}$ state tunneling splittings $\Delta_{V}$ cannot be determined from the extant fluorescence data. Alves et al. [27] published a large body of dispersed single vibronic level fluorescence spectra excited from doublet components of gaseous tropolone at room temperature by a UV laser with a $1 \mathrm{~cm}^{-1}$ band width. The dispersed fluorescence spectra access many high lying $\mathrm{S}_{0}$ state vibrations that may be basically unobservable to direct IR absorption spectroscopy. This work added greatly to understanding of the vibrational spectra in the $\mathrm{S}_{1}$ and $\mathrm{S}_{0}$ electronic states - especially concerning the lowest frequency modes.

Turning to spectral doublings observed in the IR spectra of $\operatorname{TRN}(\mathrm{OH})$ and TRN(OD), it was found that samples isolated in Ne matrices produce many more transitions than there are fundamental vibrations [24]. Of these, ten pairs of lines were originally assigned as apparent spectral doublets, but in later experimental and computational research $[34,86]$ virtually all of the extraneous lines were assignable as binary, and some ternary, overtone and combination transitions. Relative absorption intensities suggest the anharmonic resonance interactions couple many of the vibrational states, and several weak computed fundamentals of TRN $(\mathrm{OH})$ and TRN(OD) have not yet been observed in IR spectroscopic experiments. Spectral tunneling doublet assignments in the Ne matrix data were made only for the $\mathrm{OH}(\mathrm{OD})$ stretching (DS $=19[4.8] \mathrm{cm}^{-1}$ ) and skeletal contortion (DS = $10.6[9.6] \mathrm{cm}^{-1}$ ) fundamentals. The absence of other assignable tunneling doublets in the IR spectra of Ne-isolated TRN is attributed to the above noted total damping of the gas phase coherent tunneling splittings $\Delta_{V}<2.5 \mathrm{~cm}^{-1}$ by couplings to the Ne matrix [82]. In Ar matrices the peaks show shifting, broadening, and sometimes structure relative to the Ne matrix data [24]. 
Table 1.2 Calculated energy splittings $\left(\Delta_{0} \mathrm{~S}_{1}\right)$ in $\mathrm{cm}^{-1}$ for the 3D

model potentials for the Ã state of tropolone. (Table VII of Ref. [83].)

\begin{tabular}{|c|c|c|}
\hline Band & $\mathrm{CIS} / 6-31++\mathrm{C}(\mathrm{d}, \mathrm{p})$ & Exp. \\
\hline $13^{0} 14^{0}$ & 19.6 & 20 \\
\hline $13^{0} 14^{1}$ & 24.2 & 31 \\
\hline $13^{0} 14^{2}$ & 29.0 & 29 \\
\hline $13^{0} 14^{3}$ & 34.0 & \\
\hline $13^{0} 14^{4}$ & 39.3 & \\
\hline $13^{1} 14^{0}$ & 20.4 & 33 \\
\hline $13^{2} 14^{0}$ & 21.2 & \\
\hline $13^{3} 14^{0}$ & 21.9 & \\
\hline $13^{4} 14^{0}$ & 22.5 & \\
\hline $25^{0} 26^{0}$ & 24.4 & 20 \\
\hline $25^{0} 26^{1}$ & 24.2 & \\
\hline $25^{0} 26^{2}$ & 24.0 & 8 \\
\hline $25^{0} 26^{3}$ & 23.8 & \\
\hline $25^{0} 26^{4}$ & 23.6 & 6 \\
\hline $25^{0} 26^{5}$ & 23.4 & \\
\hline $25^{0} 26^{6}$ & 23.3 & 5 \\
\hline $25^{0} 26^{7}$ & 23.1 & \\
\hline $25^{0} 26^{8}$ & 22.8 & 2 \\
\hline $25^{1} 26^{0}$ & 23.9 & \\
\hline $25^{2} 26^{0}$ & 23.4 & 5 \\
\hline $25^{3} 26^{0}$ & 22.9 & \\
\hline $25^{4} 26^{0}$ & 22.5 & \\
\hline
\end{tabular}


Vibrations in the $\mathrm{S}_{0}$ state of gaseous $\mathrm{TRN}(\mathrm{OH})$ were directly addressed in the $700-3500 \mathrm{~cm}^{-1}$ region using high resolution Fourier transform infrared (FTIR) absorption spectroscopy [35] on samples at $25^{\circ} \mathrm{C}$, the sublimation pressure $\sim 0.01$ Torr, and optical path length $32 \mathrm{~m}$. Individual rotational transitions of tropolone are not resolved at a spectral resolution of $0.0025 \mathrm{~cm}^{-1}$. However, sharp Q branch peaks arising in rotational contours of the FTIR absorptions mark approximate band origins for the out-of-plane vibrations (type $\mathrm{C}$ profiles), and for vibrations with intense transition dipoles paralleling the fictive $C_{2}$ axis (type A profiles). The observed sharp Q branch spikes led to 32 reported [35] spectral tunneling doublets in the region between 700 and $950 \mathrm{~cm}^{-1}$. Additional doublets were observed at $0.1 \mathrm{~cm}^{-1}$ resolution at frequencies above $950 \mathrm{~cm}^{-1}$, but the details have not yet been reported - exclusive of the $\mathrm{CH} / \mathrm{OH}$ stretching region which is shown below to have a smooth contour with no resolved spectral doublets [35].

The $\mathrm{CCOH}$ torsion fundamental $\left(v_{22}\right)$ and two members of an associated progression of hot bands are shown in Fig. 1.4. The doublet separation for $v_{22}$ is $\mathrm{DS}_{22}$ $=\left|\Delta_{22}-\Delta_{0}\right|=0.90 \mathrm{~cm}^{-1}$. Together with $\Delta_{0}=0.974 \mathrm{~cm}^{-1}$ for the ZP state, this yields the strongly damped tunneling splitting $\Delta_{22}=0.074 \mathrm{~cm}^{-1}$ for this fundamental. The transitions in Fig. 1.4 have also been studied for the oxygen isotopomers ${ }^{16,18} \mathrm{TRN}(\mathrm{OH})$ and ${ }^{18,18} \mathrm{TRN}(\mathrm{OH})$ [39]. An analysis including the assumption that ${ }^{16,16} \Delta_{22} /{ }^{16,16} \Delta_{0}={ }^{18,18} \Delta_{22} /{ }^{18,18} \Delta_{0}$ for the oxygen isotopomer splittings in the $v_{22}$ $\mathrm{COH}$ torsion and ZP states contributes to first estimates for the isotopomer ZP tunneling splittings ${ }^{18,18} \Delta_{0}=0.865 \mathrm{~cm}^{-1}$ and ${ }^{16,18} \Delta_{0}=0.920 \mathrm{~cm}^{-1}$. The ${ }^{18} \mathrm{O}$ vibrational isotope shifts of the $v_{22}$ transitions depend on normal isotopomer reduced mass values near $1.07 \mathrm{amu}$. In contrast, the ${ }^{18} \mathrm{O}$ isotope dependence of the isotopomer tunneling splittings for $v_{22}$ correlates with the large reduced mass values of the skeletal contortion fundamental [39]. This unique isotope effect supports the role for skeletal tunneling in the tautomerization process of tropolone in the $\mathrm{S}_{0}$ electronic state.

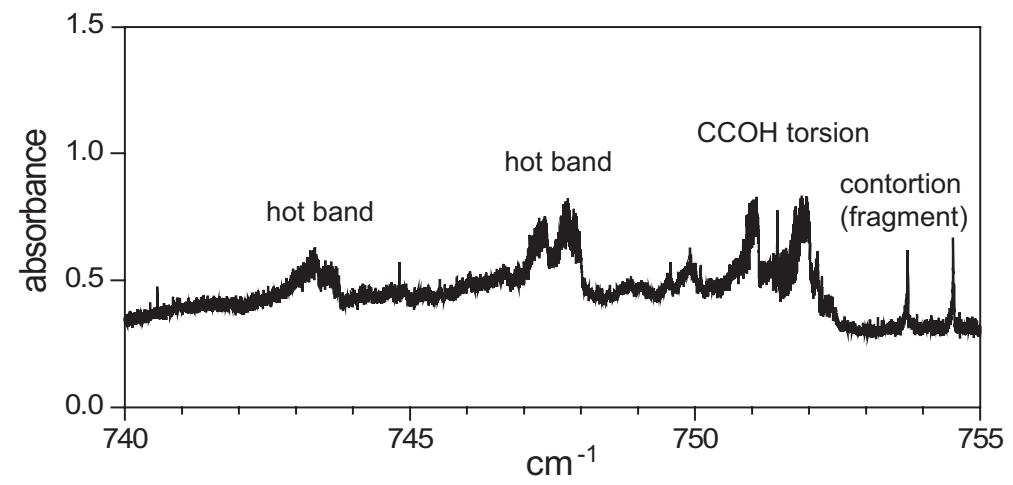

Figure 1.4 A $15 \mathrm{~cm}^{-1}$ overview of the high resolution FTIR absorption spectrum of gaseous $\operatorname{TRN}(\mathrm{OH})$ at $25^{\circ} \mathrm{C}[35]$. The spectral doublets are for the $\mathrm{CCOH}$ torsion fundamental and two members of its hot band progres- sion. The ultra-narrow spikes are attributed, with other structure, to the high frequency doublet component of the skeletal contortion fundamental. 
The $\mathrm{OH} / \mathrm{CH}$ stretching region around $3100 \mathrm{~cm}^{-1}$ is of obvious importance to a discussion of coherent $\mathrm{H}$ tunneling in TRN. Sharp Q peaks are neither predicted nor observed for the $\mathrm{OH}$ stretching fundamental, although Fig. 1.5 shows peaks appear in the region. Vibrational assignments $[34,86]$ were proposed on the basis of (i) gas phase IR spectra at lower frequencies, (ii) moderately high level MO computations, (iii) two-laser fluorescence dip IR spectra (FDIRS) of jet-cooled TRN(OH) and TRN(OD) by Frost et al. [33], (iv) the IR spectra of Ne matrix-isolated samples [24], and (v) the results of independent theoretical computations of the spectral doublet for the $\mathrm{OH}$ stretching fundamental. The weak $\mathrm{OH}$ stretching fundamental assigned in Fig. $1.5\left[3121,3102 \mathrm{~cm}^{-1}\right.$ in Ne-isolated TRN(OH)] occurs near the five $\mathrm{CH}$ stretching fundamentals (overlapped at $3063,3030 \mathrm{~cm}^{-1}$ ), with anharmonic resonance couplings to these and many (perhaps all) of the binary overtone and combination states present in the region. The broad gas phase absorption for TRN $(\mathrm{OH})$ closely spans the limits set by these transitions (as

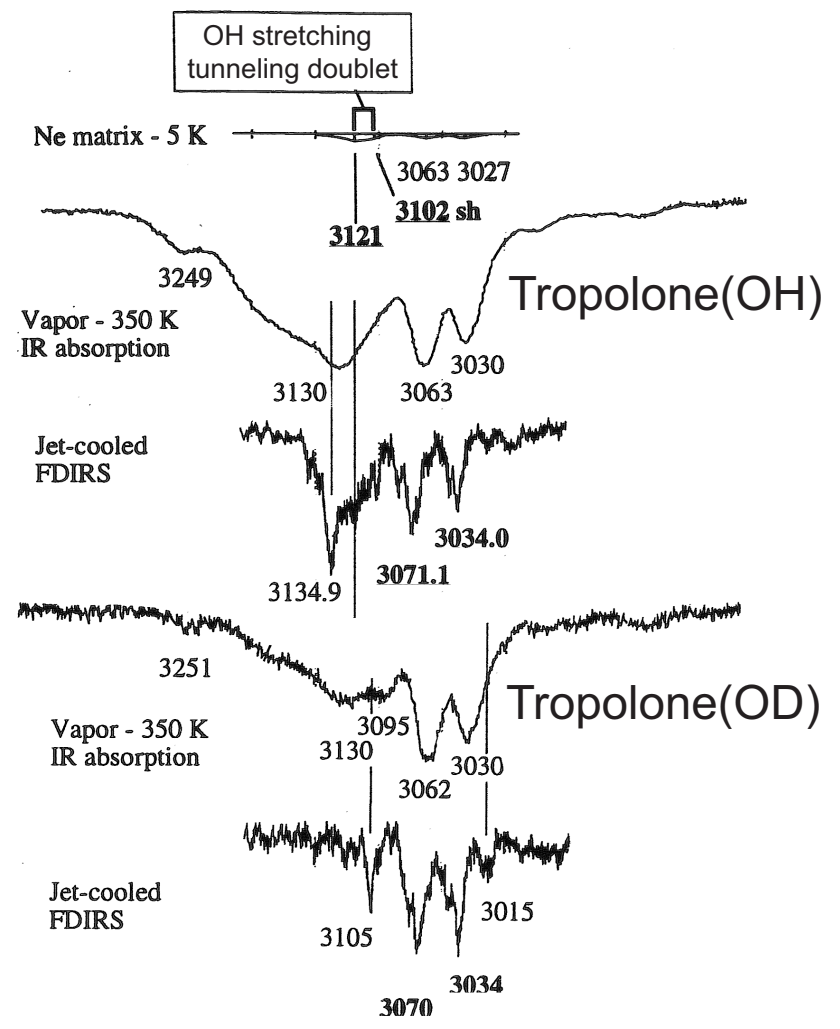

Figure 1.5 Infrared spectra of $\operatorname{TRN}(\mathrm{OH})$ and TRN (OD) in the $3100 \mathrm{~cm}^{-1}$ region [34]. In Ne-isolated TRN $(\mathrm{OH})$ the fundamental $\mathrm{OH}$ stretching spectral doublet is at $3121 \mathrm{~cm}^{-1}$

and a shoulder at $3102 \mathrm{~cm}^{-1}$. The doublet is not discerned in the gas phase IR absorption profile. The jet-cooled fluorescence dip IR spectra (FDIRS) are due to Frost et al. [33]. 
shown in Fig. 3 of Ref. [34].) Hot band absorptions are also present. The assignment of the OD stretching fundamental is 2344.8, $2340 \mathrm{~cm}^{-1}$ for Ne-isolated TRN(OD), with DS $=4.8 \mathrm{~cm}^{-1}$. Because of damping by the Ne matrix, the spectral doublet separations for gaseous TRN $(\mathrm{OH})$ and TRN(OD) would be $\sim 2 \mathrm{~cm}^{-1}$ larger than the 19 and $5 \mathrm{~cm}^{-1}$ values observed in Ne-matrix isolation [82].

As noted in Section 1.2 for MA, the vibrational state-specific tunneling dynamics of TRN are addressed using PES features obtained through quantum chemical computations. The results of computing the $C_{2 \mathrm{v}}$ saddle-point energy barrier maxima [86] for TRN at various levels of theory are shown in Table 1.3. The SP barriers computed using the larger basis sets with second order Møller-Plesset perturbation theory (MP2) for correlation energy are only about one quarter of the values obtained using the restricted Hartree-Fock (RHF) methodology with the 6$31 \mathrm{G}(\mathrm{d}, \mathrm{p})$ basis set. Vener et al. [87] addressed the problem of coherent $\mathrm{H}$ tunneling in tropolone by approximating solutions to the 2D and 3D Schrödinger equations for the $\mathrm{OH} \cdots \mathrm{O}$ group using adiabatic separations of the high and low frequency degrees of freedom. The molecular PES was constructed using the 6-31G basis with added polarization functions for some atoms. Self-consistent field (SCF) and configuration interaction singles (CIS) methodologies were used for the $\mathrm{S}_{0}$ and $\mathrm{S}_{1}$ states, respectively. The computed vibrational state-specific tunneling splittings reflect the dynamics of $\mathrm{H}$ motion in a double-minimum PES with a high barrier maximum $\left(\sim 15.7 \mathrm{kcal} \mathrm{mol}^{-1}\right)$. The computational model produced enhanced tunneling splitting on exciting the $\mathrm{O} \cdots \mathrm{O}$ stretching coordinate and modest effects on exciting $\mathrm{COH}$ bending. Tunneling behavior on the excitation of vibrations beyond the $\mathrm{OH} \cdots \mathrm{O}$ group was not considered. Smedarchina et al. [88] used a modified semiclassical instanton approach to consider the ZP and excited state tunneling splittings of the $\mathrm{S}_{0}$ and $\mathrm{S}_{1}$ states. PES features were computed at the RHF/6-31G(d,p) level. Scaled by 0.9 , the high computed adiabatic barrier reproduced the experimental $\mathrm{ZP}$ splitting for $\mathrm{S}_{1}$ tropolone, and the splittings computed for 13 excited vibrational states yielded excellent to poor agreement with experiment. The instanton computations produced a large deuterium isotope effect, as observed.

Takada and Nakamura [89] studied $\mathrm{S}_{0}$ tropolone using model 3D analytical PES functions based on coordinates for the $\mathrm{OH}$ stretch, a tunneling-promoting nominal $\mathrm{O} \cdots \mathrm{O}$ stretch, and a low frequency out-of-plane deformation mode. These were parametrized using optimized geometries for the tautomer and SP configurations computed at the MP2/6-31G(d,p) level, with refinement of the critical point energies at the MP4/6-31G(d,p) level. ZP energies for all coordinates, except the three defining the PES, were included using vibrational spectra computed at the MP2/6-31G(d,p) level. Tunneling eigenstates were evaluated by a numerical method. The high value of the $C_{2 \mathrm{v}}$ SP barrier entering the PES produced good computed agreement with the observed $\Delta_{0} \mathrm{~S}_{0}=0.974 \mathrm{~cm}^{-1}$ tunneling splitting. Enhanced tunneling splittings were computed for $\mathrm{O} \cdots \mathrm{O}$ stretching excitations, with mildly damped splittings for excitations of the out-of-plane mode. In a very similar paper, Wójcik et al. [83] used model 2D and 3D analytical PESs for TRN in the $\mathrm{S}_{1}$ electronic state that were parametrized by data computed at the CIS/6- 
Tab. 1.3 MO-computed $C_{2 v}$ saddle-point potential energies for tropolone [86].

\begin{tabular}{|c|c|c|c|}
\hline Level & Atoms & Basis & Energy (kcal mol-1) \\
\hline \multirow[t]{2}{*}{$M P 2^{[a]}$} & $\mathrm{COHOC}$ & 6-311G(df,pd) & 3.64 \\
\hline & $5 \mathrm{CH}$ & $6-311 G(d, p)$ & \\
\hline \multirow[t]{2}{*}{ MP3 } & $\mathrm{COHOC}$ & 6-311G(df,pd) & 7.12 \\
\hline & $5 \mathrm{CH}$ & $6-311 G(d, p)$ & \\
\hline \multirow[t]{2}{*}{ MP4(DQ) } & COHOC & 6-311G(df,pd) & 7.88 \\
\hline & $5 \mathrm{CH}$ & $6-311 G(d, p)$ & \\
\hline \multirow[t]{2}{*}{ MP4(SDQ) ${ }^{[b]}$} & $\mathrm{COHOC}$ & 6-311G(df,pd) & 6.96 \\
\hline & $5 \mathrm{CH}$ & $6-311 G(d, p)$ & \\
\hline \multirow[t]{2}{*}{ MP2 } & $\mathrm{C}_{7} \mathrm{OHO}$ & $6-311 G(\mathrm{df}, \mathrm{pd})$ & 3.64 \\
\hline & $5 \mathrm{H}$ & $6-311 G(d, p)$ & \\
\hline \multirow[t]{2}{*}{ MP2 } & COHOC & $6-311++G(d f, p d)$ & 3.62 \\
\hline & $5 \mathrm{CH}$ & 6-311G(d,p) & \\
\hline \multirow[t]{2}{*}{ MP2 } & $\mathrm{OHO}$ & 6-311G(2df,2pd) & 4.57 \\
\hline & $\mathrm{C}_{7} \mathrm{H}_{5}$ & $6-311 G(d, p)$ & \\
\hline MP2 & all & $6-311 G(d, p)$ & 5.02 \\
\hline MP2 & all & $6-31++G(d, p)$ & 5.57 \\
\hline RHF & all & $6-31 G(d, p)$ & 15.62 \\
\hline MP2 & all & $6-31 G(d, p)$ & 5.29 \\
\hline MP3 & all & $6-31 G(d, p)$ & 9.22 \\
\hline MP4(DQ) & all & $6-31 G(d, p)$ & 9.93 \\
\hline MP4(SDQ) & all & $6-31 G(d, p)$ & 8.86 \\
\hline
\end{tabular}

a MP2/GEN methodology is used for geometry optimizations and harmonic frequency computations.

b MP4(SDQ)/GEN methodology is used for points in the PES topography. The G3(MP2) computed SP energy is $7.2 \mathrm{kcal} \mathrm{mol}^{-1}$ [93].

$31++\mathrm{G}(\mathrm{d}, \mathrm{p})$ level of theory (including the vibrational spectrum for the ZP correction). The tunneling splittings, accurately computed using a variational method, are shown with the experimental splittings for $\mathrm{S}_{1}$ tropolone in Table 1.2 taken from the article. 
Paz et al. [90] used MO-computed energies at modest theoretical levels to parametrize a 2D model PES and obtain tunneling splittings resembling the observed data. As they did for MA, Guo et al. [91] used a semiclassical approach on the tunneling splittings, with trajectory calculations, to compute $\Delta_{V}$ values for all 39 fundamentals of the $\mathrm{S}_{0}$ state of $\operatorname{TRN}(\mathrm{OH})$. MO-computations were used to establish the PEF functions. With adjustment of the barrier to give the observed $\Delta_{0}$ value, the two lowest frequency out-of-plane modes realistically damp the tunneling, several likely modes enhance the tunneling, and the $\Delta_{\mathrm{OH}}$ for the $\mathrm{OH}$ stretch is $31 \mathrm{~cm}^{-1}$. The scope of the various developing multidimensional theories is general and, while the computations on TRN lag those on MA, inroads on higher dimension computations are being made. Giese and Kühn [92] applied a multidimensional reaction surface approach to TRN using 4D and 12D versions of the reaction surface. The 4D surface included reduced normal modes favoring $\mathrm{OH}$ stretch, $\mathrm{COH}$ bend, the skeletal mode near $750 \mathrm{~cm}^{-1}$ presented $[34,86]$ as the tautomerization coordinate, and $\mathrm{O} \cdots \mathrm{O}$ stretching. The 12D model was used to compute IR spectra using the multiconfiguration time-dependent Hartree method. The spectrum provides theoretical justification for the small $\Delta_{\mathrm{OH}}$ tunneling splitting value, and for the attribution of the broad $\mathrm{OH}$ stretching absorption of gaseous TRN to resonantly coupled binary combination modes as discussed above for Fig. 1.5. This work and investigations described above for TRN -and in the previous section for MA - give prominence to the lowest frequency in-plane mode (nominal $\mathrm{O} \cdots \mathrm{O}$ stretching) as the $\mathrm{H}$ transfer coordinate.

As already noted in Section 1.2 Tautermann et al. [71] obtained good agreement with experiment for the ZP splittings of MA. They also successfully applied their semiclassical method for finding the tunneling path to $(\mathrm{HF})_{2}$ and TRN [93], where the $7.2 \mathrm{kcal} \mathrm{mol}^{-1}$ quantum chemistry barrier for TRN is 50 to $100 \%$ lower than the barriers used for the computations on TRN discussed above. It is similar to the MP4/GEN results (footnote b of Table 1.3) used in the following discussion of the tautomerization of $\mathrm{S}_{0}$ TRN that is based on an examination of the computed and spectroscopic data set for TRN(OH) and TRN(OD) [34-36, 86]. MP2/GEN (footnote a in Table 1.3) was used for geometry optimizations and computation of harmonic vibrational spectra, with MP4(SDQ)/GEN refinement of energies at critical points and other PES points of interest. The unscaled MP2/GEN computed vibrational spectra provided insight into sorting of the fundamental vibrations of $\mathrm{TRN}(\mathrm{OH})$ and TRN(OD) from the numerous binary (and occasional ternary) overtone and combination transitions resolved in the Ne matrix-isolation IR spectra [24, 34]. Rostkowska et al. [94] recently reported a normal mode analysis for the TRN monomer, and Wójcik et al. [95] studied normal modes of the dimer along with IR and Raman spectra of polycrystalline tropolone. To interpret the observed broadening of the $\mathrm{OH}$ absorption, they applied a model coupling the low frequency inter- and intramolecular $\mathrm{O} \cdots \mathrm{O}$ stretching modes to the $\mathrm{OH}$ and $\mathrm{OD}$ stretching vibrations.

The MP2/GEN optimized minimum energy path (MEP) [86] was found to be very long and to reach $C_{2 v}$ geometry at a high energy PES bifurcation point - the source of the energy ridge that divides the tautomers and hosts $C_{2 \mathrm{v}}$ SP configura- 


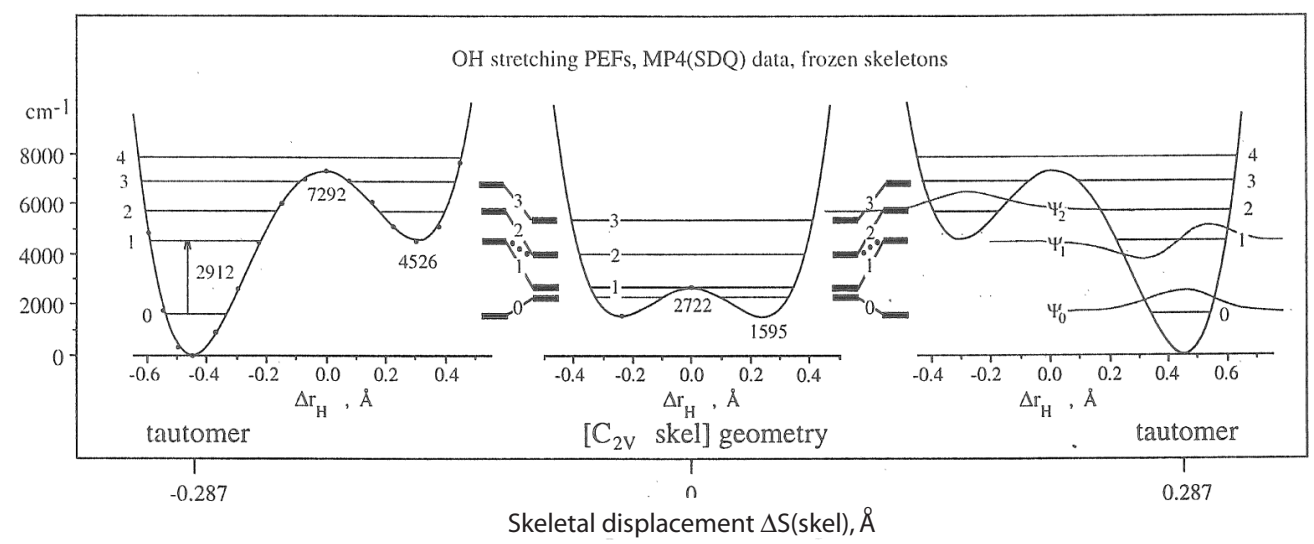

Figure 1.6 Dependence of the 1D potential energy function for $\mathrm{OH}$ stretching on the skeletal geometry [86]. PEFs are shown for the localized $\mathrm{OH}$ at the tautomer configurations, and for the delocalized $\mathrm{H}$ at the intermediate $\mathrm{C}_{2 \mathrm{v}}$ saddle-point configuration of the $\mathrm{C}_{7} \mathrm{H}_{5} \mathrm{O}_{2}$ skeleton. Plotted as a function of the skeletal displacement $\Delta S$ (skel) (see also Fig. 1.7), the $\mathrm{OH}$ stretching eigenvalues $E_{1}$ and $E_{2}$ show an avoided crossing symbolized by dots between the levels correlated in the present figure.

tions. At geometries near the tautomer minima the $\mathrm{H}$ atom is found to be localized to one $\mathrm{O}$ atom as suggested in Fig. 1.6. The figure shows that with contortion of the 14-atom $\mathrm{C}_{7} \mathrm{H}_{5} \mathrm{O}_{2}$ skeleton to $\mathrm{C}_{2 \mathrm{v}}$ configurations on the energy ridge the $\mathrm{OH}$ stretching PEF is transformed into an equal double-minimum function allowing full delocalization of the $\mathrm{H}$ atom. The contortional displacement coordinate thus "vibrationally assists" $\mathrm{H}$ tunneling in $\mathrm{S}_{0}$ tropolone which, in turn, allows completion of the skeletal tautomerization. The first examinations of this dynamical model are being made at the lowest possible descriptive level: informal adiabatic separation of the fast $\mathrm{OH}$ stretching and slow skeletal contortion motions, with quasiharmonic separation of the other 37 vibrations. Despite the disregarded kinetic and potential energy couplings, the informally separated 1D equations allow key features of the tunneling behavior to be outlined and compared with experimental data.

The quasiharmonic vibration spectrum is computed at the MP2/GEN level. The 1D OH stretching PEF (Fig. 1.6) is computed using MP4/GEN input points and its vibrational energies are solved numerically. The 1D PEF for the contortion is given quadratic-quartic-Gaussian functionality anchored by MP4/GEN level critical point energies supplemented with the 37 MP2/GEN computed quasiharmonic ZP or excited vibrational energies - and the numerically calculated, geometry-dependent, $\mathrm{OH}$ stretching vibrational energy. The Gaussian functionality of the PEF interpolates the vibrational energy contribution between the SP and tautomer geometries. The effective PEF for the tautomerization coordinate depends on the other-coordinate vibrational state. The state-specific ZP contortion levels in the upper and lower vibrational states of the spectroscopic transitions give first estimates for the spectral doublet separations as illustrated for an out-of-plane mode $v_{\mathrm{X}}$ in Fig. 1.7. 


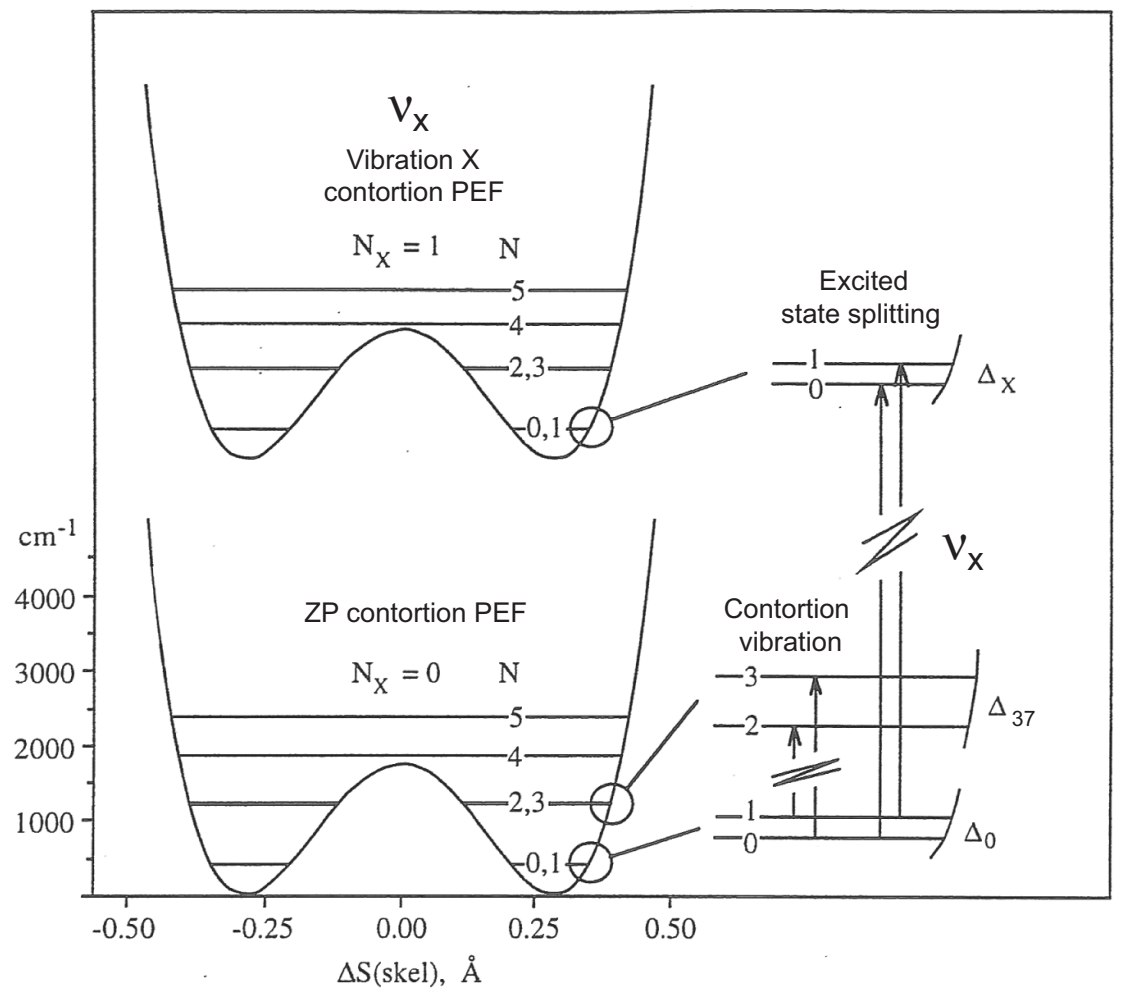

Figure 1.7 Vibrational state-specific effective potential energy functions for the skeletal contortion vibration [86]. Transitions at 754 , $743.3 \mathrm{~cm}^{-1}$ for TRN $(\mathrm{OH})$ isolated in a $\mathrm{Ne}$ matrix are assigned as the spectral doublet, and $\Delta_{37}$ is the upper state splitting, of the $v_{37}$

contortion vibration. The spectral doublet for a vibration $v_{x}$, for example the $\mathrm{CCOH}$ torsion in Fig. 1.4, has the upper state vibrational state-specific tunneling splitting $\Delta_{\mathrm{x}}$. The spectral doublet components have the separation $\mathrm{DS}_{\mathrm{x}}=\left|\Delta_{\mathrm{x}}-\Delta_{0}\right|$.

The model [34-36, 39, 86], labeled the \{tunneling skeleton\}\{tunneling $\mathrm{H}$ atom [TSTH] model for convenience, closely approximates the frequencies and doublet separations of the contortion fundamental as observed in Ne matrix isolation at 754, $743.4 \mathrm{~cm}^{-1}$ for TRN(OH) and 751, $741.4 \mathrm{~cm}^{-1}$ for TRN(OD). Peaks assigned to the higher - but not the lower - frequency component are discerned in the complex IR absorption profiles of gaseous TRN $(\mathrm{OH})$. The observed ${ }^{18} \mathrm{O}$ isotope shifts, $3.3 \mathrm{~cm}^{-1}$ for ${ }^{16,18 \mathrm{TRN}}(\mathrm{OH})$ and $6.9 \mathrm{~cm}^{-1}$ for ${ }^{18,18} \mathrm{TRN}(\mathrm{OH})$, are reproduced by the TSTH model. The MP2/GEN isotope shifts for the $754 \mathrm{~cm}^{-1}$ transition, if treated as a quasiharmonic mode, are about twice these values. The contortional reduced mass values are determined as $16,16 \mu=7.25,16,18 \mu=7.32$, and $18,18 \mu=$ $7.37 \mathrm{amu}$, and these values also correlate the observed ${ }^{18} \mathrm{O}$ effects on the tunneling splittings for the $\mathrm{COH}$ torsion fundamental shown for ${ }^{16,16} \mathrm{TRN}(\mathrm{OH})$ in Fig. 1.4. The TSTH model approximates the frequencies and small tunneling splittings of the $\mathrm{OH}(\mathrm{OD})$ stretching fundamentals observed in Ne matrix isolation at 3121, 
$3102 \mathrm{~cm}^{-1}$ for TRN(OH) and 2344.8, $2340 \mathrm{~cm}^{-1}$ for TRN(OD). The successful computation of the $\mathrm{OH}$ and $\mathrm{OD}$ stretching frequencies depends on a curve-hopping at the major avoided crossing point lying in plots of the $\mathrm{OH}$ and $\mathrm{OD}$ stretching energies versus contortional displacement. Additional support for the TSTH model is discussed in the articles [34-36, 39, 86]. Spectroscopic experiments pending for tropolone include comprehensive high resolution FTIR spectra for TRN(OD) and its ${ }^{18} \mathrm{O}$ isotopomers - plus extension of the spectral range to the $300 \mathrm{~cm}^{-1}$ region for all TRN isotopomers.

\section{4}

\section{Tropolone Derivatives}

Sekiya and his collaborators [44-48] found spectral doublets in the $\mathrm{S}_{1}-\mathrm{S}_{0}$ excitation and dispersed fluorescence spectra of jet-cooled halotropolones that are modifications of the basic coherent tunneling splittings observed for $\operatorname{TRN}(\mathrm{OH})$ and TRN(OD). The symmetrical chlorotropolones showed enhancements of the spec-

Tab. 1.4 Tunneling splittings $\left(\mathrm{cm}^{-1}\right)$ in the chlorotropolones and bromotropolones [48].

\begin{tabular}{|c|c|c|c|c|}
\hline \multirow[t]{2}{*}{ Molecule } & \multicolumn{4}{|c|}{$\left|\Delta_{v^{\prime}}-\Delta_{0^{\prime \prime}}\right|$} \\
\hline & $0_{0}^{0}$ & $26_{0}^{2}$ & $26_{0}^{4}$ & $14_{0}^{1}$ \\
\hline $\mathrm{TRN}(\mathrm{OH})$ & 19 & 7 & 4 & 30 \\
\hline 5-chloro-TRN $(\mathrm{OH})$ & 23 & 4 & $\leq 1$ & 21 \\
\hline 3,7-dichloro-TRN(OH) & 45 & 7 & $\leq 1$ & 53 \\
\hline 3,5,7-trichloro-TRN $(\mathrm{OH})$ & 31 & 25 & 12 & 38 \\
\hline 5-bromo-TRN(OH) & 16 & 6 & 3 & $13^{[a]}$ \\
\hline 3,7-dibromo-TRN(OH) & $\leq 1$ & & & \\
\hline TRN(OD) & 2 & $\leq 1$ & $\leq 1$ & 11 \\
\hline 5-chloro-TRN(OD) & 2 & $\leq 1$ & & \\
\hline 3,7-dichloro-TRN(OD) & 8 & 1 & $\leq 1$ & 6 \\
\hline 3,5,7-trichloro-TRN(OD) & 4 & 2 & 2 & 6 \\
\hline 5-bromo-TRN(OD) & 2 & $\leq 1$ & & \\
\hline 3,7-dibromo-TRN(OD) & $\leq 1$ & & & \\
\hline
\end{tabular}

a This $14_{0}{ }^{1}$ transition is only tentatively assigned. 
tral doublets for the $\mathrm{S}_{1}-\mathrm{S}_{0}$ origin bands, while the bromotropolones showed damping. Spectral doublets are absent for the jet-cooled asymmetrical molecules because there is a large asymmetry offset of the energy minima of the double-well PESs [47]. Observations for the ZP, out-of-plane deformation, and nominal O $\cdots O$ stretching spectral doublets are shown in Table 1.4 [48]. The less pronounced consequences on the effective PESs, vibrational spectral doublets, and symmetries arising through the deuteration of the 3-, 4-, and 5- positions have been presented $[41,96]$.

The couplings between the $\mathrm{OH} \cdots \mathrm{O}$ and $-\mathrm{NH}_{2}$ groups of the 5-amino-tropolone molecule have been of experimental and theoretical interest [52]. In part this is because the $-\mathrm{NH}_{2}$ in the $\mathrm{S}_{0}$ state may be pyramidal, and in the $\mathrm{S}_{1}$ state it may be planar. The 5-hydroxy-tropolone molecule is planar with an asymmetric doubleminimum PES, and part of its interest resides in the possibility of driving the tropolone tautomerization by laser pumping of the $\mathrm{C}(5) \mathrm{OH}$ torsion vibration $[49,50$, 97]. In the lowest energy configuration the two $\mathrm{OH}$ bonds point in opposing directions. Resolved spectral tunneling structures similar to those of the parent TRN $(\mathrm{OH})$ and TRN(OD) molecules are observed for the aforementioned molecules, as well as others: 5-methyl-tropolone [53, 54], isopropyltropolones [55], and 5-phenyl-tropolone [56-57]. The effects of coupling the $\mathrm{OH} \cdots \mathrm{O}$ coordinates to the methyl internal rotation, or of coupling them to the low frequency phenyl torsional motions, are of interest.

\section{5}

\section{Concluding Remarks}

The zero-point tunneling splittings of $\mathrm{S}_{0}$ malonaldehyde, $\Delta_{0}=21.583 \mathrm{~cm}^{-1}$, and $\mathrm{S}_{0}$ tropolone, $\Delta_{0}=0.974 \mathrm{~cm}^{-1}$, plus values for $\mathrm{S}_{0} \mathrm{MA}$ isotopomers, are accurately known. The ZP splitting for TRN $(\mathrm{OH})$ in the $\widetilde{\mathrm{A}}^{1} \mathrm{~B}_{2}\left(\pi^{*}-\pi\right), \mathrm{S}_{1}$, state is $19.846 \mathrm{~cm}^{-1}$ and that for $\mathrm{MA}(\mathrm{OH})$ in the $\widetilde{\mathrm{A}}^{1} \mathrm{~B}_{1}\left(\pi^{*}-\mathrm{n}\right), \mathrm{S}_{1}$, state is $\sim 2.5 \mathrm{~cm}^{-1}$. A sizeable array of less precise vibrational state specific tunneling splittings is known for $S_{0}$ and $\mathrm{S}_{1} \mathrm{TRN}(\mathrm{OH})$. For $\mathrm{S}_{0} \mathrm{MA}(\mathrm{OH})$ this type of experimental evidence includes the value $\Delta_{6}=21.55 \mathrm{~cm}^{-1}$ for $v_{6}$ at $1594 \mathrm{~cm}^{-1}$, several small doublet separations $\mathrm{DS}_{\mathrm{V}} \sim 0 \mathrm{~cm}^{-1}$ implying $\Delta_{V} \sim \Delta_{0}$ in the midrange region, and doublet separations $\mathrm{DS}_{V}=\left|\Delta_{V}-\Delta_{0}\right|$ of several $\mathrm{cm}^{-1}$ for each of the four fundamentals below $600 \mathrm{~cm}^{-1}$. Spectral evidence clearly defining the splitting behavior and couplings for $\mathrm{H}$ motions in the OHO group of $\mathrm{MA}(\mathrm{OH})$ have not yet been recognized. The IR spectrum of $\mathrm{S}_{0}$ TRN shows clear-cut tunneling doublets in the region below $950 \mathrm{~cm}^{-1}$, evidence for small splittings in the midfrequency range, and DS = $19(5) \mathrm{cm}^{-1}$ for $\mathrm{OH}(\mathrm{OD})$ stretching. All in all, the evidence supporting state-specific coherent tunneling behavior in these molecules is very strong and future experimental work can be expected to provide many accurately measured data points fully amenable to discriminating interpretive models and the discovery of novel intramolecular phenomena. Experimental effort, including overtone and combination spectra, clarifying the dynamical behavior of the OHO modes is a priority 
need. The ZP $\Delta_{0}$ values for the $\mathrm{S}_{0} \mathrm{MA}$ and TRN isotopomers are reasonably reproduced by several different semiclassical and $a b$ initio quantum chemical methods - in some cases remarkably well. The extant work shows the ZP tunneling splittings are very sensitive to the quality of the utilized $a b$ initio input PES, and the scatter of results among the method-dependent computed splitting behavior verifies this is true for all energy states. The developments for coordinate systems and coordinate transformations for the kinetic and potential energy operators are well advanced and several full (21D) dimensional computations have been published for MA. The theoretical models use low-dimensioned reaction surface analysis for the large amplitude motions. PES expansions for the remaining orthogonalized, presumably tunneling inert, "bath" modes are truncated at the quadratic, and for some models the quartic, terms. It is important to have accurately computed energy values at distances well out from the expansion points, and the "Morsification" of the stretching coordinate expansions is an approach under examination [98]. Strong promoting modes for $\mathrm{H}$ tunneling are found to be sparse. The $\mathrm{OH}$ stretching PEF for $\mathrm{S}_{0}$ TRN shown in Fig. 1.6 is an example with pronounced behavior of a type proposed for consideration of an enzyme-substrate complex [99]. Effects of the strong resonance couplings arising in some PES versions [61] are duly noted.

\section{Acknowledgments}

The author is very grateful for the help of T. E. Redington with this chapter. 


\section{References}

1 Y. Cha, C. J. Murray, J. P. Klinman, Science 1989, 243, 1325.

2 T. Jonsson, M. H. Glickman, S. J. Sun, J. P. Klinman, J. Am. Chem. Soc. 1996, 118, 10319.

3 A. Kohen, R. Cannio, S. Bartolucci, J. P. Klinman, Nature 1999, 399, 496.

4 M. J. Knapp, J. P. Klinman, Eur. J. Biochem. 2002, 269, 3113.

5 J. Basran, M. J. Sutcliffe, N. S. Scrutton, Biochemistry 1999, 38, 3218.

6 M. J. Sutcliffe, N. S. Scrutton, Eur. J. Biochem. 2002, 269, 3096.

7 D. Antoniou, S. Caratzoulas, C. Kalyanaraman, J. S. Mincer, S. D. Schwartz, Eur. J. Biochem. 2002, 269, 3103.

8 P. S. Zuev, R. S. Sheridan, T. V. Albu, D. G. Truhlar, D. A. Hrovat, W. T. Borden, Science 2003, 299, 867.

9 W. F. Rowe Jr., R. W. Duerst, E. B. Wilson, J. Am. Chem. Soc. 1976, 98, 4021.

10 S. L. Baughcum, R. W. Duerst, W. F. Rowe, Z. Smith, E. B. Wilson, J. Am Chem. Soc. 1981, 103, 6296.

11 P. Turner, S. L. Baughcum, S. L. Coy, Z. Smith, J. Am. Chem. Soc. 1984, 106, 2265.

12 S. L. Baughcum, Z. Smith, E. B. Wilson, R. W. Duerst, J. Am. Chem. Soc. 1984, 106, 2260.

13 D. W. Firth, K. Beyer, M. A. Dvorak, S. W. Reeve, A. Grushow, K. R. Leopold, J. Chem. Phys. 1991, 94, 1812.

14 T. Baba, T. Tanaka, I. Morino, K. M. T. Yamada, K. Tanaka, J. Chem. Phys. 1999, 110, 4131.

15 Z. Smith, E. B. Wilson, R. W. Duerst, Spectrochim. Acta, Part A 1983, 39, 1117.

16 C. J. Seliskar, R. E. Hoffman, J. Mol. Spectrosc. 1982, 96, 146.

17 D. W. Firth, P. F. Barbara, H. P. Trommsdorff, Chem. Phys. 1989, 136, 349.

18 T. Chiavassa, P. Roubin, L. Pizzala, P. Verlaque, A. Allouche, F. Marinelli, J. Phys. Chem. 1992, 96, 10659.

19 C. Duan, D. Luckhaus, Chem. Phys. Lett. 2004, 391, 129.
20 C. J. Seliskar, R. E. Hoffman, J. Mol. Spectrosc. 1981, 88, 30.

21 A. A. Arias, T. A. W. Wasserman, P. H. Vaccaro, J. Chem. Phys. 1997, 107, 5617.

22 A. C. P. Alves, J. M. Hollas, Mol. Phys. 1972, 23, 927.

23 A. C. P. Alves, J. M. Hollas, Mol. Phys. 1973, 25, 1305.

24 R. L. Redington, T. E. Redington, J. Mol. Spectrosc. 1979, 78, 229.

25 R. Rossetti, L. E. Brus, J. Chem. Phys. 1980, 73, 1546.

26 Y. Tomioka, M. Ito, N. Mikami, J. Phys. Chem. 1983, 87, 4401.

27 A. C. P. Alves, J. M. Hollas, H. Musa, T. Ridley, J. Mol. Spectrosc. 1985, 109, 99.

28 R. L. Redington, Y. Chen, G. J. Scherer, R. W. Field, J. Chem. Phys. 1988, 88, 627.

29 R. L. Redington, R. W. Field, Spectrochim. Acta, Part A 1989, 45, 41.

30 H. Sekiya, Y. Nagashima, Y. Nishimura, Bull. Chem. Soc. Jpn. 1989, 62, 3229.

31 H. Sekiya, Y. Nagashima, Y. Nishimura, J. Chem. Phys. 1990, 92, 5761.

32 K. Tanaka, H. Honjo, T. Tanaka, H. Kohguchi, Y. Ohshima, Y. Endo, J. Chem. Phys. 1999, 110, 1969.

33 R. K. Frost, F. C. Hagemeister, C. A. Arrington, T. S. Zwier, K. D. Jordan, J. Chem. Phys. 1996, 105, 2595.

34 R. L. Redington, T. E. Redington, J. M. Montgomery, J. Chem. Phys. 2000, 113, 2304.

35 R. L. Redington, R. L. Sams, J. Phys. Chem. A 2002, 106, 7494.

36 R. L. Redington, R. L. Sams, Chem. Phys. 2002, 283, 135.

37 A. E. Bracamonte, P. H. Vaccaro, J. Chem. Phys. 2003, 119, 997.

38 A. E. Bracamonte, P. H. Vaccaro, J. Chem. Phys. 2004, 120, 4638.

39 R. L. Redington, T. E. Redington, T. A. Blake, R. L. Sams, T. J. Johnson, J. Chem. Phys. 2005, 122, 224311.

40 H. Sekiya, Y. Nagashima, Y. Nishimura, Chem. Phys. Lett. 1989, 160, 581.

41 H. Sekiya, Y. Nagashima, T. Tsuji, Y. Nishimura, A. Mori, H. Takeshita, J. Phys. Chem. 1991, 95, 10311. 
42 H. Sekiya, K. Sasaki, Y. Nishimura, Z.-H. Li, A. Mori, H. Takeshita, Chem. Phys. Lett. 1990, 173, 285.

43 R. L. Redington, T. E. Redington, M. A. Hunter, R. W. Field, J. Chem. Phys. 1990, 92, 6456.

44 H. Sekiya, K. Sasaki, Y. Nishimura, A. Mori, H. Takeshita, Chem. Phys. Lett. 1990, 174, 133.

45 T. Tsuji, H. Sekiya, Y. Nishimura, A. Mori, H. Takeshita, J. Chem. Phys. 1991, 95, 4801.

46 T. Tsuji, H. Sekiya, Y. Nishimura, R. Mori, A. Mori, H. Takeshita, J. Chem. Phys. 1992, 97, 6032.

47 T. Tsuji, H. Sekiya, S. Ito, H. Ujita, M. Habu, A. Mori, H. Takeshita, Y. Nishimura, J. Chem. Phys. 1993, 98, 6571.

48 H. Sekiya, T. Tsuji, S. Ito, A. Mori, H. Takeshita, Y. Nishimura, J. Chem. Phys. 1994, 101, 3464.

49 F. A. Ensminger, J. Plassard, T. S. Zwier, S. Hardinger, J. Chem. Phys. 1995, 102, 5260.

50 R. K. Frost, F. Hagemeister, D. Schleppenbach, G. Laurence, T. S. Zwier, J. Phys. Chem. 1996, 100, 16835.

51 T. Tsuji, H. Hamabe, Y. Hayashi, H. Sekiya, A. Mori, Y. Nishimura, J. Chem. Phys. 1999, 110, 966.

52 F. A. Ensminger, J. Plassard, T. S. Zwier, J. Phys. Chem. 1993, 97, 4344.

53 K. Nishi, H. Sekiya, H. Kawakami, A. Mori, Y. Nishimura, J. Chem. Phys.1998, 109, 1589.

54 K. Nishi, H. Sekiya, H. Kawakami, A. Mori, Y. Nishimura, J. Chem. Phys. 1999, 111, 3961.

55 H. Sekiya, H. Takesue, Y. Nishimura, Z-H. Li, A. Mori, H. Takeshita, J. Chem. Phys. 1990, 92, 2790.

56 T. Tsuji, Y. Hayashi, H. Sekiya, H. Hamabe, Y. Nishimura, H. Kawakami, A. Mori, Chem. Phys. Lett. 1997, 278, 49.

57 T. Tsuji, Y. Hayashi, H. Hamabe, H. Kawakami, A. Mori, Y. Nishimura, H. Sekiya, J. Chem. Phys. 1999, 110, 8485.

58 P. R. Bunker, P. Jensen, Molecular Symmetry and Spectroscopy, 2nd Edn., NRC Research Press, Ottawa 1998.
59 S. F. Tayyari, F. Milani-Nejad, Spectrochim. Acta, Part A, 1998, 54, 255.

60 A. Alparone, S. Millefiori, Chem. Phys. 2003, 290, 15.

61 V. A. Benderskii, E. V. Vetoshkin, I. S. Irgibaeva, H. P. Trommsdorff, Chem. Phys. 2000, 262, 393.

62 T. D. Sewell, Y. Guo, D. L. Thompson, J. Chem. Phys. 1995, 103, 8557.

63 R. Meyer, T.-K Ha, Mol. Phys. 2003, 101, 3263.

64 D. P. Tew, N. C. Handy, S. Carter, Mol. Phys. 2004, 102, 2217.

65 T. Carrington, W. H. Miller, J. Phys. Chem. 1986, 84, 4364.

66 N. Shida, P. F. Barbara, J. E. Almlöf, J. Chem. Phys. 1989, 91, 4061.

67 K. Yagi, G. V. Mil'nikov, T. Taketsugu, K. Hirao, H. Nakamura, Chem. Phys. Lett. 2004, 397, 435.

68 Z. Smedarchina, W. Siebrand, M. Z. Zgierski, J. Chem. Phys. 1995, 103, 5326.

69 K. Giese, O. Kühn, J. Chem. Phys. 2004, 120, 4107.

70 N. Došlic, O. Kühn, Z. Phys. Chem. 2003, 217, 1507.

71 C. S. Tautermann, A. F. Voegele, T. Loerting, K. R. Liedl, J. Chem. Phys. 2002, 117, 1962.

72 V. Barone, C. Adamo, J. Chem. Phys. 1996, 105, 11007.

73 S. Sadhukhan, D. Muñoz, C. Adamo, G. E. Scuseria, Chem. Phys. Lett. 1999, 306, 83.

74 K. Yagi, T. Taketsugu, K. Hirao, J. Chem. Phys. 2001, 115, 10647.

75 N. Makri, W. H. Miller, J. Chem. Phys. 1989, 91, 4026.

76 M. D. Coutinho-Neto, A. Viel, U. Manthe, J. Chem. Phys. 2004, 121, 9207.

77 G. V. Mil'nikov, H. Nakamura, J. Chem. Phys. 2001, 115, 6881.

78 G. V. Mil'nikov, K. Yagi, T. Taketsugu, H. Nakamura, K. Hirao, J. Chem. Phys. 2004, 120, 5036.

79 D. P. Tew, N. C. Handy, S. Carter, S. Irle, J. Bowman, Mol. Phys. 2003, 101, 2513.

80 L. M. Jackman, J. C. Trewella, R. C. Haddon, J. Am. Chem. Soc. 1980, 102, 2519.

81 N. Makri, W. H. Miller, J. Chem. Phys. 1987, 86, 1451. 
82 R. L. Redington, T. E. Redington, J. Chem. Phys. 2005, 122, 124304.

83 M. J. Wójcik, H. Nakamura, S. Iwata, T. Wiktor, J. Chem. Phys. 2000, 112, 6322

84 H. Shimanouchi, Y. Sasada, Acta. Crystallogr. Sect. B, 1973, 29, 81.

85 H. F. Hameka, J. R. de la Vega, J. Am. Chem. Soc. 1984, 106, 7703.

86 R. L. Redington, J. Chem. Phys. 2000, 113, 2319.

87 M. V. Vener, S. Scheiner, N. D. Sokolov, J. Chem. Phys. 1994, 101, 9755.

88 Z. Smedarchina, W. Siebrand, M. Z. Zgierski, J. Chem. Phys. 1996, 104, 1203.

89 S. Takada, H. Nakamura, J. Chem. Phys. 1995, 102, 3977.

90 J. J. Paz, M. Moreno, J. M. Lluch, J. Chem. Phys. 1995, 103, 353.
91 Y. Guo, T. D. Sewell, D. L. Thompson, J. Phys. Chem. A 1998, 102, 5040.

92 K. Giese, O. Kühn, preprint.

93 C. S. Tautermann, A. F. Voegele, T. Loerting, K. R. Liedl, J. Chem. Phys. 2002, 117, 1967.

94 H. Rostkowska, L. Lapinski, M. J. Nowak, L. Adamowicz, Int. J. Quant. Chem. 2002, 90, 1163.

95 M. J. Wójcik, M. Boczar, M. Stoma, Int. J. Quant. Chem. 1999, 73, 275.

96 J. J. Paz, M. Moreno, J. M. Lluch, Chem. Phys. 1999, 246, 103.

97 J. J. Nash, T. S. Zwier, K. S. Jordan, J. Chem. Phys. 1995, 102, 5360.

98 R. Burcl, S. Carter, N. C. Handy, Chem. Phys. Lett. 2003, 373, 357.

99 A. Kohen, J. P. Klinman, Chem. Biol. 1999, 6, R191. 
